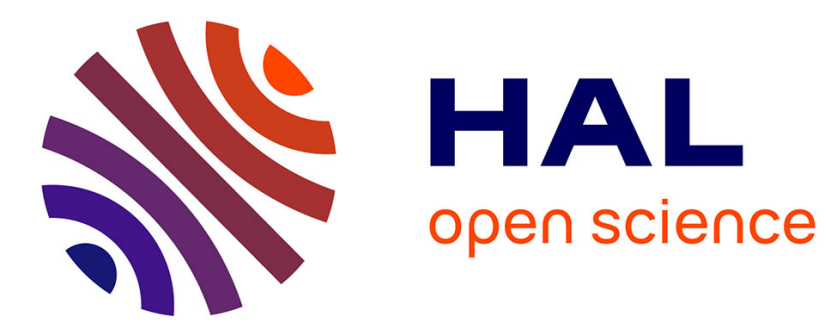

\title{
A framework for the control of nonholonomic mobile manipulators
}

\author{
Matthieu Fruchard, Pascal Morin, Claude Samson
}

\section{To cite this version:}

Matthieu Fruchard, Pascal Morin, Claude Samson. A framework for the control of nonholonomic mobile manipulators. [Research Report] RR-5556, INRIA. 2005, pp.52. inria-00070450

\section{HAL Id: inria-00070450 \\ https://hal.inria.fr/inria-00070450}

Submitted on 19 May 2006

HAL is a multi-disciplinary open access archive for the deposit and dissemination of scientific research documents, whether they are published or not. The documents may come from teaching and research institutions in France or abroad, or from public or private research centers.
L'archive ouverte pluridisciplinaire HAL, est destinée au dépôt et à la diffusion de documents scientifiques de niveau recherche, publiés ou non, émanant des établissements d'enseignement et de recherche français ou étrangers, des laboratoires publics ou privés. 


\title{
A framework for the control of nonholonomic mobile manipulators
}

\author{
Matthieu Fruchard — Pascal Morin — Claude Samson
}

$\mathbf{N}^{\circ} 5556$

April 2005

Thème NUM 



\title{
A framework for the control of nonholonomic mobile manipulators*
}

\author{
Matthieu Fruchard, Pascal Morin, Claude Samson \\ Thème NUM — Systèmes numériques \\ Projets Icare
}

Rapport de recherche $\mathrm{n}^{\circ} 5556$ - April 2005 - 52 pages

\begin{abstract}
We propose a general framework for the feedback control of nonholonomic mobile manipulators. The control laws derived with this approach allow to simultaneously execute a manipulation task and monitor the situation (position and orientation) of the nonholonomic platform on which the manipulator is mounted. The active monitoring of the platform's situation significantly augments the manipulation capabilities of the system. For instance, the manipulation objective is still achievable when it compels the platform to move backward or perform manœuvres. This monitoring is also instrumental for the avoidance of the manipulator's joint limits.
\end{abstract}

Key-words: mobile manipulator, nonholonomic system, task-function, transverse function.

* This work has been supported by the French program ROBEA. 


\section{Une méthodologie pour la commande des manipulateurs mobiles non-holonomes ${ }^{\dagger}$}

Résumé : Nous proposons une méthodologie de synthèse de retours d'état pour les manipulateurs mobiles non-holonomes. Les lois de commande obtenues à partir de cette approche permettent d'exécuter une tâche de manipulation et, dans le même temps, de contrôler la situation (i.e. position et orientation) de la plateforme mobile qui sert de support au bras manipulateur. Le contrôle actif de la situation de la plateforme augmente les capacités du système de façon significative. En particulier, il permet d'assurer la réalisation de la tâche de manipulation même lorsque celleci nécessite, de la part de la platforme mobile, des mouvements en marche arrière ou des manœuvres. C'est également un élément essentiel pour traiter le problème d'évitement des butées articulaires du bras manipulateur.

Mots-clés : manipulateur mobile, système non-holonome, fonction de tâche, fonction transverse.

$\dagger$ Ce travail a été soutenu par le programme national ROBEA. 


\section{Contents}

1 Introduction 4

2 Notation and preliminary recalls $\quad 6$

2.1 Group operation in $S E(2) \ldots \ldots \ldots \ldots \ldots$

2.2 Kinematic relations . . . . . . . . . . . . . . . 8

2.3 Transverse functions and omnidirectional companion frame . . . . 10

3 Control approach $\quad 11$

3.1 Control problem statement . . . . . . . . . . . . . . 12

3.2 Guidelines for the choice of the primary task-function . . . . . . . . 15

3.3 Guidelines for the choice of the secondary cost-function and the control of the mobile platform . . . . . . . . . . . . . 17

3.3.1 Secondary cost-function . . . . . . . . . . . . . 17

3.3.2 Control of the mobile platform . . . . . . . . . . 18

4 Application examples $\quad \mathbf{2 2}$

4.1 Target tracking in $\mathrm{SE}(2) \ldots \ldots \ldots . \ldots . \ldots . \ldots 22$

4.1.1 The manipulation task and associated control . . . . . . . 23

4.1.2 Secondary cost-function and control of the mobile platform . 24

4.1 .3 Simulation results . . . . . . . . . . . . . . 27

4.2 A tracking application in $\mathbb{R}^{2} \ldots \ldots \ldots \ldots . \ldots . \ldots . \ldots 39$

4.2.1 Manipulation task and associated control . . . . . . . . 39

4.2.2 Secondary cost function and control of the platform . . . . . 40

4.2 .3 Simulation results . . . . . . . . . . . . . 41

5 Extension to more general mobile manipulators 46

$\mathrm{RR} \mathrm{n}^{\circ} 5556$ 
Fruchard, Morin, \& Samson

\section{Introduction}

A mobile manipulator, composed of a manipulator arm mounted on a mobile platform, is far more versatile than a conventional manipulator whose base is fixed, as a consequence of its enlarged operational space. The diversity of robotic devices of this kind (see e.g. [3] for a survey) reflects how this latter feature can be exploited in various ways.

The present paper is devoted to the feedback control of mobile manipulators and, more specifically, to the control of mobile manipulators whose platform is subjected to nonholonomic kinematic constraints, e.g. unicycle-like or car-like platforms. From now on, such devices will, for short, be referred to as nonholonomic mobile manipulators. While the platform's motion and the manipulation task can sometimes be performed separately, i.e. one after the other, there are also applications for which motion coordination between the two subsystems is necessary. The main difficulty of controlling a nonholonomic mobile manipulator, by comparison to the case of a classical manipulator arm, or a manipulator mounted on an omnidirectional platform, comes from the kinematic constraints which limit the platform's motion capabilities. In general, these constraints do not prevent the platform from being controllable, and an important literature has been devoted to the design of open-loop controls for trajectory planning purposes (see e.g. [5, 7, 13, 3]). However, Brockett's theorem [4] implies that the asymptotic stabilization of a nonholonomic platform at a specified fixed configuration cannot be obtained by using smooth (or even continuous) purestate feedback. This result has important implications. For instance, it points out the impossibility, with classical feedbacks, to control the position and the orientation of a nonholonomic vehicle simultaneously. While several techniques have been developed in the last fifteen years for the stabilization of nonholonomic systems, via more general types of feedbacks (see e.g. [11] for a survey), the utilization of these techniques for mobile manipulators has seldom been explored. The methods proposed so far in the literature typically rely on the partial control of the platform's situation. For instance, in [18], the unicycle-like platform is controlled in position via input-output linearization techniques, in [15, 2] some (two at most) combinations of position and orientation variables are controlled to a desired value via the selection of associated independent "tasks", in [17] the controller is obtained via Lagrange necessary conditions for a constrained optimization problem. These techniques find their justification in the fact that, for some applications, it is not necessary to control both the position and orientation of the platform. Such is the case when, for example, the manipulation task does not require the platform to perform manœuvres (i.e. forward/backward transitions), and/or when no joint limit severely reduces the 
manipulator's operational space. However, for other applications, these methods will fail because they cannot prevent the occurrence of the so-called "knife-edge" effect (for car-like platforms for example), or the collision with joint limits. Controlling the platform in both position and orientation is necessary to avoid these problems.

The present paper aims at presenting a control design framework in view of executing a manipulation task and, at the same time, stabilizing/controlling the situation of the supporting nonholonomic platform. The realization of the manipulation task is set as the prime objective. As such, it usually requires a precise positioning of the manipulator's end-effector. By contrast, such a precision is seldom necessary (or even desirable) for the positioning of the platform, because small errors at this level can be compensated by using the manipulator's degrees of freedom. For this reason, the control objective for the platform is here expressed in the form of a secondary cost whose exact minimization is not a strict requirement. A nominal role devoted to this secondary minimization is to bring the platform to a domain where the manipulation task can be successfully carried out. The proposed approach relies on a previous work by two of the authors on the stabilization of nonholonomic systems, and more specifically on the transverse function (t.f.) approach $[9,10]$. The key element is the possibility to conceptually associate an omnidirectional companion frame with a frame characterizing the situation of a nonholonomic vehicle. The property conveyed by the transverse function used to define this companion frame is that the vehicle's frame is bound to stay close to its companion, whatever the motion of the latter frame. Moreover, the distance between the two frames can be modified at will, and rendered arbitrarily small via the choice of the transverse function parameters. In order to control the vehicle, it then suffices to control the omnidirectional companion frame, a much easier task. This approach exploits the strong controllability properties of nonholonomic systems, and especially the fact that any (not necessarily admissible) smooth trajectory of situations can be approximately followed, with arbitrarily good precision, by a nonholonomic (controllable) mobile robot $[6,16]$. While studies in this direction have already been conducted in the context of trajectory planning, we are not aware of similar attempts in the context of nonholonomic mobile manipulators and (reactive) feedback control.

The paper is organized as follows. We recall in Section 2 some classical relations concerning the kinematic modeling of mobile manipulators, and a few results concerning the transverse function approach. The general control framework is presented in Section 3. Application examples are given in Section 4, and illustrated through simulation results. To simplify the exposition, the presentation of the framework in Sections 2, 3, and 4, is done for the sub-class of planar manipulators mounted

$\mathrm{RR} \mathrm{n}^{\circ} 5556$ 
on unicycle-like platforms. However, the control approach extends mutatis mutandis when the end-effector of the manipulation arm evolves in the three-dimensional cartesian space. Due to the generality of the t.f. approach, the present method also applies to many other nonholonomic platforms, with very minor modifications. This point is discussed in more details in Section 5, and illustrated through the example of a car-like platform.

\section{Notation and preliminary recalls}

Let us first introduce some notation. $I_{n}$ is the identity matrix associated with $\mathbb{R}^{n}$. $R(\theta), S$, and $\bar{R}(\theta)$, are the rotation matrices defined by:

$$
R(\theta)=\left(\begin{array}{cc}
\cos \theta & -\sin \theta \\
\sin \theta & \cos \theta
\end{array}\right), \quad S=R(\pi / 2)=\left(\begin{array}{cc}
0 & -1 \\
1 & 0
\end{array}\right), \quad \bar{R}(\theta)=\left(\begin{array}{cc}
R(\theta) & 0 \\
0 & 1
\end{array}\right)
$$

The following notation is used to describe the configuration of the planar mobile manipulator depicted on Fig. 1.

- $q$ is the vector of either prismatic or revolute joint coordinates of the manipulator arm. Its dimension is $n_{q}$.

- $\mathcal{F}_{0}=\left\{O, \overrightarrow{\imath_{0}}, \overrightarrow{\jmath_{0}}\right\}$ is a fixed frame in the plane.

- $\mathcal{F}_{b}=\left\{B, \overrightarrow{\imath_{b}}, \overrightarrow{\jmath_{b}}\right\}$ is a frame attached to the mobile platform in the plane. If the mobile platform is of the unicycle type (and thus nonholonomic), we impose that the instantaneous velocity of the point $B$ w.r.t. (with respect to) $\mathcal{F}_{0}$ is along the unit vector $\overrightarrow{\imath_{b}}$.

- $\mathcal{F}_{e}=\left\{E, \overrightarrow{\imath_{e}}, \overrightarrow{\jmath_{e}}\right\}$ is a frame attached to the manipulator's end-effector.

- Given two arbitrary frames $\mathcal{F}_{a}=\left\{A, \overrightarrow{\imath_{a}}, \overrightarrow{\jmath_{a}}\right\}$ and $\mathcal{F}_{b}=\left\{B, \overrightarrow{\imath_{b}}, \overrightarrow{\jmath_{b}}\right\}$, the "situation" of $\mathcal{F}_{b}$ w.r.t. $\mathcal{F}_{a}$ is defined as the element $r_{a b} \in S E(2)=\mathbb{R}^{2} \times S^{1}$ (with $S^{1}=\mathbb{R} / 2 \pi \mathbb{Z}$ ) given by

$$
r_{a b}=\left(\begin{array}{c}
p_{a b} \\
\theta_{a b}
\end{array}\right)
$$

with $p_{a b}=\left(x_{a b}, y_{a b}\right)^{T}$ the components of $\overrightarrow{A B}$ in the basis of $\mathcal{F}_{a}$, and $\theta_{a b}$ the oriented angle between $\overrightarrow{\imath_{a}}$ and $\overrightarrow{\imath_{b}}$. When $\mathcal{F}_{a}$ is the fixed frame $\mathcal{F}_{0}$ defined 
above, $r_{a b}=r_{0 b}$ is simply called the "situation" of $\mathcal{F}_{b}$, and the index 0 is omitted, i.e.

$$
r_{0 b}=r_{b}=\left(\begin{array}{c}
p_{b} \\
\theta_{b}
\end{array}\right)
$$

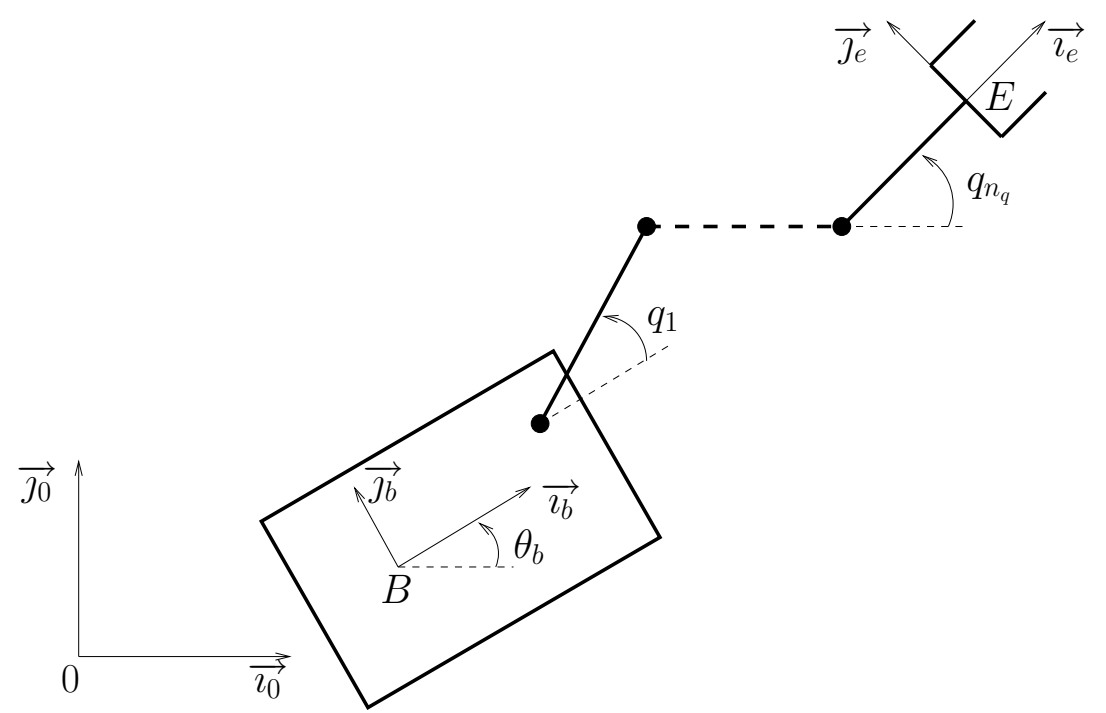

Figure 1: Planar mobile manipulator

\subsection{Group operation in $S E(2)$}

The space of frames in the plane is isomorphic to the Lie group $S E(2)$. With the above notation, a possible isomorphism between these two spaces is given by the mapping $\mathcal{F}_{b} \longmapsto r_{b}$. Furthermore, it is also well known that the mapping

$$
\left(\begin{array}{l}
p \\
\theta
\end{array}\right) \longmapsto\left(\begin{array}{cc}
R(\theta) & p \\
0 & 1
\end{array}\right)
$$

with $R(\theta)$ the $2 \times 2$ rotation matrix of angle $\theta$ in the plane, defines a Lie group isomorphism between $S E(2)$ and the matrix Lie group of $3 \times 3$ homogeneous matrices. On this latter group, the group product is just given by the matrix product, i.e.

$$
\left(\begin{array}{cc}
R\left(\theta_{1}\right) & p_{1} \\
0 & 1
\end{array}\right)\left(\begin{array}{cc}
R\left(\theta_{2}\right) & p_{2} \\
0 & 1
\end{array}\right)=\left(\begin{array}{cc}
R\left(\theta_{1}+\theta_{2}\right) & p_{1}+R\left(\theta_{1}\right) p_{2} \\
0 & 1
\end{array}\right)
$$

RR $\mathrm{n}^{\circ} 5556$ 
and, accordingly, the group operation on $S E(2)$ is defined by

$$
\left(\begin{array}{l}
p_{1} \\
\theta_{1}
\end{array}\right)\left(\begin{array}{c}
p_{2} \\
\theta_{2}
\end{array}\right)=\left(\begin{array}{c}
p_{1}+R\left(\theta_{1}\right) p_{2} \\
\theta_{1}+\theta_{2}
\end{array}\right)
$$

The identity element $e$ on $S E(2)$, and the inverse $r^{-1}$ of $r=(p, \theta)^{T}$ (such that $r r^{-1}=r^{-1} r=e$ ) are therefore given by

$$
e=\left(\begin{array}{l}
0 \\
0
\end{array}\right) \quad \text { and } \quad r^{-1}=\left(\begin{array}{c}
-R(-\theta) p \\
-\theta
\end{array}\right)=-\bar{R}(-\theta) r
$$

Since the group operation plays the same role as the addition in the case of $\mathbb{R}^{n}$, a "difference" between two elements of $S E(2)$ is given by the product of one element by the inverse of the other one (and thus not by a component-to-component substraction). For instance, a difference between $r_{e}$ and $r_{p}$ is $r_{p e}=\left(r_{p}\right)^{-1} r_{e}=r_{p 0} r_{0 e}$; let us recall that $r_{p e}$ is the situation of the frame $\mathcal{F}_{e}$ w.r.t. the frame $\mathcal{F}_{p}$. Note that, due to the non-commutativity of the group operation, the product order is important, and one can form several "differences" between two elements of $S E(2)$. For example, $r_{e p}=\left(r_{e}\right)^{-1} r_{p}=r_{e 0} r_{0 p}=\left(r_{p e}\right)^{-1}$ characterizes the situation of the frame $\mathcal{F}_{p}$ w.r.t. $\mathcal{F}_{e}$. A possible distance between two frames $\mathcal{F}_{a}$ and $\mathcal{F}_{b}$ is given by

$$
\begin{aligned}
d\left(\mathcal{F}_{a}, \mathcal{F}_{b}\right):=\mathrm{d}_{\gamma}\left(r_{a}, r_{b}\right) & :=\sqrt{\left\|p_{a}-p_{b}\right\|^{2}+2 \gamma\left(1-\cos \left(\theta_{a}-\theta_{b}\right)\right)} \\
& =\sqrt{\left\|p_{a b}\right\|^{2}+2 \gamma\left(1-\cos \theta_{a b}\right)}
\end{aligned}
$$

with $\gamma>0$, and $\|$.$\| the euclidean norm (on \mathbb{R}^{2}$ in the present case). Note that the last equality in (2) indicates that $\mathrm{d}_{\gamma}$ is a left-invariant distance on $S E(2)$, i.e. $\mathrm{d}_{\gamma}\left(r_{a}, r_{b}\right)=\mathrm{d}_{\gamma}\left(e, r_{a b}\right)$.

\subsection{Kinematic relations}

The kinematic model of the manipulator arm is simply given by $\dot{q}=u_{q}$. Let us now consider the mobile platform. The components of the velocity of the point $B$ w.r.t. $\mathcal{F}_{0}$, expressed in $\mathcal{F}_{b}$, are denoted as $v_{b, 1}$ and $v_{b, 2}$, i.e.

$$
\frac{d \overrightarrow{O B}}{d t}=v_{b, 1} \overrightarrow{\imath_{b}}+v_{b, 2} \overrightarrow{\jmath b}
$$

The angular velocity of the frame $\mathcal{F}_{b}$ w.r.t. $\mathcal{F}_{0}$ is denoted as $\omega_{b}$, i.e.

$$
\frac{d \theta_{b}}{d t}=\omega_{b}
$$


By setting $u_{b}:=\left(v_{b, 1}, v_{b, 2}, \omega_{b}\right)$, one easily verifies that

$$
\dot{r}_{b}=\bar{R}\left(\theta_{b}\right) u_{b}
$$

Obviously, a similar relation holds not only for $\mathcal{F}_{b}$ but also for any other frame. Following [12], $u_{b}$ is called the "body velocity of the frame $\mathcal{F}_{b}$ ".

Let $r_{c}$ and $r_{d}$ denote two smooth curves on $S E(2)$ with $\dot{r}_{c}=\bar{R}\left(\theta_{c}\right) u_{c}$ and $\dot{r}_{d}=$ $\bar{R}\left(\theta_{d}\right) u_{d}$. The following relation, which can be verified by direct calculation from (1) and (3), is well known (see e.g. [12, Prop. 2.15]):

$$
\stackrel{\cdot}{r_{c} r_{d}}=\bar{R}\left(\theta_{c}+\theta_{d}\right)\left(u_{d}+\operatorname{Ad}_{r_{d}^{-1}} u_{c}\right)
$$

with $\mathrm{Ad}_{r^{-1}}$ the matrix defined by the following relations:

$$
\operatorname{Ad}_{r}=\left(\begin{array}{cc}
R(\theta) & -S p \\
0 & 1
\end{array}\right), \quad \operatorname{Ad}_{r^{-1}}=\left(\mathrm{Ad}_{r}\right)^{-1}=\left(\begin{array}{cc}
R(-\theta) & R(-\theta) S p \\
0 & 1
\end{array}\right)
$$

From the above relations and the fact that $r^{-1} r=e$, one deduces that for any smooth curve $r$ on $S E(2)$ with $\dot{r}=\bar{R}(\theta) u$,

$$
\stackrel{\cdot}{r^{-1}}=\bar{R}(-\theta)\left(-\operatorname{Ad}_{r} u\right)
$$

Finally, by combining (4) and (6), one also obtains:

$$
\overbrace{r_{c} r_{d}^{-1}}^{\cdot}=\bar{R}\left(\theta_{c}-\theta_{d}\right) \operatorname{Ad}_{r_{d}}\left(u_{c}-u_{d}\right)
$$

and

$$
\overbrace{r_{c}^{-1} r_{d}}^{\cdot}=\bar{R}\left(\theta_{d}-\theta_{c}\right)\left(u_{d}-\operatorname{Ad}_{r_{d}^{-1} r_{c}} u_{c}\right)
$$

which may also be written as

$$
\dot{r}_{c d}=\bar{R}\left(\theta_{c d}\right) u_{c d}, \quad \text { with } \quad u_{c d}=u_{d}-\operatorname{Ad}_{r_{d c}} u_{c}
$$

Let $h:(q, r, t) \longmapsto h(q, r, t)$ denote a differentiable function from $\mathbb{R}^{n(q)} \times S E(2) \times$ $\mathbb{R}$ to $\mathbb{R}^{p}$. With some abuse of notation, we denote by $\frac{\partial h}{\partial r}$ the function such that, for any smooth curves $(q, r)($.$) on \mathbb{R}^{n(q)} \times S E(2)$,

$$
\frac{d}{d t} h(q(t), r(t), t)=\frac{\partial h}{\partial q}(q(t), r(t), t) \dot{q}(t)+\frac{\partial h}{\partial r}(q(t), r(t), t) u(t)+\frac{\partial h}{\partial t}(q(t), r(t), t)
$$

with $\dot{r}=\bar{R}(\theta) u$, and $\frac{\partial h}{\partial q}$ and $\frac{\partial h}{\partial t}$ the (standard) partial derivatives of $h$ w.r.t. $q$ and $t$.

$\mathrm{RR} \mathrm{n}^{\circ} 5556$ 


\subsection{Transverse functions and omnidirectional companion frame}

Consider a smooth function

$$
f: \alpha \longmapsto f(\alpha)=\left(\begin{array}{c}
p_{f}(\alpha) \\
\theta_{f}(\alpha)
\end{array}\right)
$$

from $S^{1}$ to $S E(2)$. Then,

$$
\bar{r}_{b}(\alpha):=r_{b} f(\alpha)^{-1}=\left(\begin{array}{c}
p_{b}-R\left(\theta_{b}-\theta_{f}(\alpha)\right) p_{f}(\alpha) \\
\theta_{b}-\theta_{f}(\alpha)
\end{array}\right)=\left(\begin{array}{c}
\bar{p}_{b} \\
\bar{\theta}_{b}
\end{array}\right)
$$

belongs to $S E(2)$ and, as such, represents the situation of some frame $\mathcal{F}_{\bar{b}}(\alpha)$, the origin of which has components

$$
-R\left(-\theta_{f}(\alpha)\right) p_{f}(\alpha)
$$

in the frame $\mathcal{F}_{b}$. With the distance between $\mathcal{F}_{b}$ and $\mathcal{F}_{\bar{b}}(\alpha)$ defined by (2), one obtains:

$$
d_{\gamma}\left(\mathcal{F}_{b}, \mathcal{F}_{\bar{b}}(\alpha)\right)=\mathrm{d}_{\gamma}\left(r_{b}, \bar{r}_{b}(\alpha)\right)=\mathrm{d}_{\gamma}\left(r_{b}, r_{b}(f(\alpha))^{-1}\right)=\mathrm{d}_{\gamma}\left(e,(f(\alpha))^{-1}\right)=\mathrm{d}_{\gamma}(f(\alpha), e)
$$

Therefore,

$$
\forall \alpha \in S^{1}, \quad d_{\gamma}\left(\mathcal{F}_{b}, \mathcal{F}_{\bar{b}}(\alpha)\right) \leqslant \sup _{\alpha} \mathrm{d}_{\gamma}(f(\alpha), e)=\sqrt{p_{f}(\alpha)^{2}+\gamma\left(1-\cos \theta_{f}(\alpha)\right)}
$$

By using (7), one easily obtains that the kinematics of $\mathcal{F}_{\bar{b}}(\alpha)$ is given by:

$$
\dot{\bar{r}}_{b}=\bar{R}\left(\bar{\theta}_{b}\right) \bar{u}_{b}
$$

with

$$
\bar{u}_{b}:=\left(\begin{array}{cc}
I_{2} & -S p_{f}(\alpha) \\
0 & 1
\end{array}\right)\left(\bar{R}\left(\theta_{f}(\alpha)\right)-\frac{\partial f}{\partial \alpha}(\alpha)\right)\left(\begin{array}{c}
u_{b} \\
\dot{\alpha}
\end{array}\right)
$$

the body velocity of the frame $\mathcal{F}_{\bar{b}}(\alpha)$. Note that the argument $\alpha$ of $\bar{r}_{b}(\alpha)$ in (12) has been omitted to lighten the notation. From now on, $\dot{\alpha}$ will be viewed as a complementary control input to monitor the motion of the frame $\mathcal{F}_{\bar{b}}(\alpha)$. The important point is that the frame $\mathcal{F}_{\bar{b}}(\alpha)$ is omnidirectional provided that $\bar{u}_{b}$ can be rendered equal to any vector in $\mathbb{R}^{3}$ (i.e. provided that the mapping $\left(u_{b}, \dot{\alpha}\right) \longmapsto \bar{u}_{b}$ is onto). When the mobile platform is omnidirectional, this property is satisfied for any function $f$. For example, one can take $f=0$, in which case $\mathcal{F}_{b}$ and $\mathcal{F}_{\bar{b}}(\alpha)$ coincide. The 
case of nonholonomic mobile platforms is more interesting. Consider a unicycle-like platform. Then, the second component of $u_{b}$ (i.e. $v_{b, 2}$ ) is equal to zero, so that:

$$
\bar{u}_{b}=\left(\begin{array}{cc}
I_{2} & -S p_{f}(\alpha) \\
0 & 1
\end{array}\right) H(\alpha)\left(\begin{array}{c}
v_{b, 1} \\
\omega_{b} \\
\dot{\alpha}
\end{array}\right)=\bar{H}(\alpha)\left(\begin{array}{c}
v_{b, 1} \\
\omega_{b} \\
\dot{\alpha}
\end{array}\right)
$$

with

$$
H(\alpha):=\left(\begin{array}{ccr}
\cos \theta_{f}(\alpha) & 0 & -\frac{\partial x_{f}}{\partial \alpha}(\alpha) \\
\sin \theta_{f}(\alpha) & 0 & -\frac{\partial y_{f}}{\partial \alpha}(\alpha) \\
0 & 1 & -\frac{\partial \theta_{f}}{\partial \alpha}(\alpha)
\end{array}\right) \quad \text { and } \quad \bar{H}(\alpha):=\left(\begin{array}{cc}
I_{2} & -S p_{f}(\alpha) \\
0 & 1
\end{array}\right) H(\alpha)
$$

Therefore, $\mathcal{F}_{\bar{b}}(\alpha)$ is omnidirectional provided that the matrix $H(\alpha)$ is invertible. A function $f$ for which this property is satisfied for any $\alpha \in S^{1}$ is called a "transverse function" [9]. The issue of the existence of such functions has been treated in the much more general context of the transverse function approach $[9,10]$. In the present case, a family of transverse functions is given by:

$$
f(\alpha)=\left(\begin{array}{c}
\varepsilon_{1} \sin \alpha \\
\varepsilon_{1} \varepsilon_{2} \frac{\sin 2 \alpha}{4} \\
\arctan \left(\varepsilon_{2} \cos \alpha\right)
\end{array}\right) \quad \text { with } \quad \varepsilon_{1}, \varepsilon_{2}>0
$$

Indeed, one can verify that for any $\alpha \in S^{1}, \operatorname{det} H(\alpha)=-\frac{\varepsilon_{1} \varepsilon_{2}}{2} \cos \left(\arctan \left(\varepsilon_{2} \cos \alpha\right)\right)<$ 0 .

Definition 1 Given a transverse function $f$ and some $\alpha \in S^{1}$, the corresponding omnidirectional frame $\mathcal{F}_{\bar{b}}(\alpha)$ associated with the nonholonomic frame $\mathcal{F}_{b}$ is called a companion frame of $\mathcal{F}_{b}$.

From what precedes, whatever the motion of $\mathcal{F}_{\bar{b}}(\alpha)$, the distance between $\mathcal{F}_{b}$ and $\mathcal{F}_{\bar{b}}(\alpha)$ never exceeds $\sup _{\alpha} \mathrm{d}_{\gamma}(f(\alpha), e)$, a bound which can be made arbitrarily small (but different from zero) via the choice of $\varepsilon_{1}$ and $\varepsilon_{2}$ in (16). Therefore, a way of controlling $\mathcal{F}_{b}$ consists in controlling its companion frame $\mathcal{F}_{\bar{b}}(\alpha)$, a much easier task since $\mathcal{F}_{\bar{b}}(\alpha)$ is omnidirectional.

\section{Control approach}

Prior to addressing the control design issue itself, one must specify control objectives in relation to the tasks which the mobile manipulator is meant to perform. In this

$\mathrm{RR} \mathrm{n}^{\circ} 5556$ 
respect, it is useful to have in mind the respective roles played by the manipulator, on one hand, and the supporting mobile platform, on the other hand. In a typical application, a tool or a sensor is mounted on the manipulator's end-effector in order to perform a task involving precise movements inside a limited domain of application. We will call this task the "manipulation task". In many cases, it is preferable that the mobile platform stays motionless during the execution of the manipulation task. There are several reasons for this, such as the imperfection of the filtering of perturbations resulting from moving on an uneven terrain, or energy saving. However, moving the supporting platform may also be useful, or even become necessary, when the size of the manipulator's operational space is not sufficient to cover the range required to complete the manipulation task on a large object, or when this object moves and tends to leave the region reachable by the manipulator's end-effector.

The general idea is that the prime role of the mobile platform is to displace the manipulator so as to compensate for its limited domain of operation, in such a way that the manipulation task can be carried out without interruption. Clearly, this objective is looser than the one set on the manipulator, and it also points out a kind of "brain/legs" or "master/slave", natural decoupling and hierarchy between the two mechanical subsystems.

\subsection{Control problem statement}

A way to translate the above considerations in general mathematical terms, reminiscent of a classical approach developed for the treatment of redundancy in the case of manipulators mounted on a fixed support (see e.g. [14] for instance), consists in considering the problem of minimizing a secondary cost function, via the control of the mobile platform, under the constraint of enforcing a set of "virtual linkages" which characterize the manipulation task, via the control of the manipulator. More precisely, we will assume that the overall control objective can be loosely ${ }^{1}$ stated as follows given the duration $T$ of the task execution:

$\mathcal{P}_{0}: \quad \forall t \in[0, T], \quad$ Minimize $h_{s}\left(r_{b}, t\right)$ under the constraint that $e_{m}\left(q, r_{b}, t\right)=0$

with $e_{m}$ and $h_{s}$ being accessible to measurement (or combined measurement and estimation). The vector-valued function $e_{m}$ characterizes the manipulation task. It obviously depends on the manipulator's joint coordinates $q$. Its dependence upon

\footnotetext{
${ }^{1}$ Since the variables $q$ and $r_{b}$ vary smoothly with time, Problem $\mathcal{P}_{0}$ cannot be solved instantaneously (except, of course, when the initial conditions $r_{b}(0)$ and $q(0)$ are solutions to this problem). Therefore, $\mathcal{P}_{0}$ should only be viewed as an "asymptotic" objective pursued through the definition of $u_{b}$ and $u_{q}$.
} 
the mobile platform's situation $r_{b}$ comes from that this platform is also the base of the manipulator. The independent time-variable $t$ is used to parameterize the possible evolution of the manipulation objective, as well as possible variations of the environment (resulting, for instance, from the motion of an object/target tracked by the end-effector). The secondary objective concerning the minimization of $h_{s}$ is usually less demanding than the manipulation task because, in many cases, the "exact" minimization of this function is not a strict requirement.

When the mobile platform is holonomic, so that the mobile manipulator can be viewed as an extended manipulator arm with $\operatorname{dim}(q)+\operatorname{dim}\left(r_{b}\right)$ degrees of freedom, Problem $\mathcal{P}_{0}$ can be solved under relatively mild assumptions upon $h_{s}$ and $e_{m}$. For example, if $h_{s}\left(r_{b}, t\right)$ admits a unique minimum $r_{*}(t)$ for each $t$, and if the function $(q, t) \longmapsto e_{m}\left(q, r_{*}(t), t\right)$ is admissible [14] so that there exists a solution $q_{*}(t)$ to the equation $e_{m}\left(q, r_{*}(t), t\right)=0$, Problem $\mathcal{P}_{0}$ is solved by any control law $\left(u_{q}, u_{b}\right)$ which ensures the convergence of $\left(q(t), r_{b}(t)\right)$ to the ideal trajectory $\left(q_{*}(t), r_{*}(t)\right)$. Since the mapping $\left(u_{q}, u_{b}\right) \longmapsto\left(\dot{q}, \dot{r}_{b}\right)$ is onto in this case, the existence of a smooth ideal trajectory is sufficient to ensure the existence of a feedback control which asymptotically stabilizes this trajectory. See, for instance, [14, Chap. 4]) for the determination of such control laws from the measurement of $e_{m}$ and $h_{s}$, and the calculation of their derivatives.

In the case of a nonholonomic platform, the situation is significantly more complicated. The difficulty is twofold: i) the ideal trajectory $r_{*}(t)$ associated with the minimization of the secondary cost $h_{s}$ has no reason of being feasible for the nonholonomic platform, and ii) even if this trajectory were feasible, the design of a feedback control law capable of ensuring its asymptotic stabilization would still be problematic, due to the non-existence of universal stabilizers for these systems, as pointed out in [4], [8]. For these reasons, Problem $\mathcal{P}_{0}$ cannot constitute, as such, a suitable formulation for the control objectives. It is too restrictive in the sense that it does not underlie the existence of a control solution. In order to circumvent the abovementionned difficulties, we propose to replace Problem $\mathcal{P}_{0}$ by the following one, which applies equally well to the omnidirectional and nonholonomic cases:

$$
\mathcal{P}: \quad \forall t \in[0, T], \quad \text { Minimize } h_{s}\left(\bar{r}_{b}, t\right) \text { under the constraint that } e_{m}\left(q, r_{b}, t\right)=0
$$

with $\bar{r}_{b}$, given by (11), the situation of the companion frame $\mathcal{F}_{\bar{b}}(\alpha)$ associated with $\mathcal{F}_{b}$ (for some transverse function). Since $\mathcal{F}_{\bar{b}}(\alpha)$ is omnidirectionnal, $\bar{r}_{b}$ is not subjected to nonholonomic constraints, and Problem $\mathcal{P}$ can be addressed with the techniques developed for the control of manipulator arms. This yields the following two-step approach for the control design.

$\mathrm{RR} \mathrm{n}^{\circ} 5556$ 
Step 1: Selection of the the manipulation task $e_{m}$ and determination of $u_{q}$.

Ideally, $e_{m}$ should be chosen such that the constraint $e_{m}\left(q, r_{b}, t\right)=0$ is equivalent to the realization of the manipulation objective. In practice however, some precautions must be taken in order to avoid the explosion of the control at the crossing of jacobian singularities, and also give the priority to the avoidance of joint limits over the execution of the manipulation task. We propose to select $e_{m}$ on the basis of the following rules:

1. $e_{m}$ is $n_{q}$-dimensional and the square "jacobian" matrix $\frac{\partial e_{m}}{\partial q}\left(q, r_{b}, t\right)$ is invertible for any $(q, t)$, and any $r_{b}$ in some "large" domain $\mathcal{D}_{r}(t)$,

2. keeping all components of $e_{m}$ bounded ensures that the manipulator's joints are away from their limits,

3. keeping all components of $e_{m}$ equal to zero is equivalent to the perfect realization of the manipulation objective, provided that $\left(q(t), r_{b}(t)\right)$ belongs to some domain $\mathcal{O}_{q}(t) \times \mathcal{O}_{r}(t)$.

Rule 1 is inspired by the property of $\rho$-admissibility introduced in the context of the task-function approach [14]. In particular, the invertibility of the jacobian matrix $\frac{\partial e_{m}}{\partial q}\left(q, r_{b}, t\right)$ allows to derive control laws $u_{q}$ for the arm, well defined as long as $r_{b}$ remains in $\mathcal{D}_{r}(t)$, that ensure the convergence of $e_{m}$ to zero. With the notation of Section 2.2, such a control law is, for example, defined by

$$
u_{q}=\left(\frac{\partial e_{m}}{\partial q}\left(q, r_{b}, t\right)\right)^{-1}\left(G e_{m}\left(q, r_{b}, t\right)-\frac{\partial e_{m}}{\partial r_{b}}\left(q, r_{b}, t\right) u_{b}-\frac{\partial e_{m}}{\partial t}\left(q, r_{b}, t\right)\right)
$$

with $G$ denoting a Hurwitz-stable matrix. Indeed, the application of this control yields the closed-loop equation $\dot{e}_{m}=G e_{m}$ and, therefore, the exponential convergence of $e_{m}$ to zero. This convergence, combined with Rule 2, guarantees the avoidance of the joint limits. Finally, Rule 3 expresses that the regulation of $e_{m}$ to zero must be coherent with the realization of the manipulation objective.

Step 2: Selection of the secondary cost $h_{s}$.

In view of Rules 1 and 3 , the objective is to select $h_{s}$ such that its minimization implies that $r_{b}(t)$ belongs to $\mathcal{D}_{r}(t)$ (so that $u_{q}$ is well defined), and possibly also inside $\mathcal{O}_{r}(t)$ (so that the manipulation objective can be achieved). For example, if $\mathcal{D}_{r}(t)$ is an open set which varies continuously with time, a possible choice consists in defining $h_{s}$ by $h_{s}\left(\bar{r}_{b}, t\right)=\left(\mathrm{d}_{\gamma}\left(\bar{r}_{b}, r_{*}(t)\right)\right)^{2}$, with $r_{*}(t) \in \mathcal{D}_{r}(t)$ representing a desired situation for the mobile platform at time $t$. In this case, the "minimization" of $h_{s}$ is 
equivalent to $\bar{r}_{b}(t)=r_{*}(t)$ and, since the distance between $\bar{r}_{b}$ and $r_{b}$ is bounded by a value which can be made arbitrarily small (through the choice of the transverse function), one can guarantee in this way that the distance between $r_{b}(t)$ and $r_{*}(t)$ is always small.

We now discuss in more details possible choices for $e_{m}$ and $h_{s}$.

\subsection{Guidelines for the choice of the primary task-function}

The objective is to define some $n_{q}$-dimensional task-function $e_{m}$ satisfying Rules $1-3$ of the previous section. A possible approach consists in setting

$$
e_{m}\left(q, r_{b}, t\right)=\left(\frac{\partial h}{\partial q}\left(q, r_{b}, t\right)\right)^{T}
$$

with $h$ denoting a smooth positive cost function whose minimization w.r.t. $q$ is equivalent to zeroing its derivative $\frac{\partial h}{\partial q}$. For example, if $h$ is a strictly convex function of $q$ for any $\left(r_{b}, t\right)$, it has a unique minimum $q_{*}\left(r_{b}, t\right)$, and $e_{m}\left(q, r_{b}, t\right)=0$ if and only if $q=q_{*}\left(r_{b}, t\right)$. Then, the asymptotic stabilization of $e_{m}$ to zero is equivalent to keeping $h$ minimal. A possibility consists in choosing $h$ as the sum of three cost functions

$$
h\left(q, r_{b}, t\right)=h_{p}\left(q, r_{b}, t\right)+h_{\ell}(q, t)+h_{r}(q, t)
$$

The function $h_{p}$ is associated with Rule 3 . It must be equal to zero if and only if the manipulation objective is perfectly achieved. Take, for instance

$$
h_{p}\left(q, r_{b}, t\right)=\frac{1}{2}\left\|e_{p}\left(q, r_{b}, t\right)\right\|^{2}
$$

with $e_{p}$ a smooth vector-function the zeroing of which corresponds to enforcing the virtual linkages associated with the manipulation objective.

The cost function $h_{\ell}$ is associated with Rule 2, i.e. it is there to ensure that the joint limits cannot be reached as long as $e_{m}$ remains bounded. This function should thus grow unbounded when any of the $q_{i}$ 's tends to a corresponding joint limit. On the other hand, in view of Rule 3 , it is desirable that this cost does not affect the realization of the manipulation objective (i.e. the vanishing of $h_{p}$ ) when the $q_{i}$ 's are far from their limits. Let $q_{i}^{-}, q_{i}^{+}\left(i=1, \ldots, n_{q}\right)$ denote the lower and upper limits for the joint variable $q_{i}$, we propose

$$
h_{\ell}(q)=\frac{1}{2} \sum_{i=1}^{n_{q}} \alpha_{i} f_{i}^{2}(q) \quad\left(\alpha_{i}>0\right)
$$

$\mathrm{RR} \mathrm{n}^{\circ} 5556$ 
with $f_{i}\left(i=1, \ldots, n_{q}\right)$ real-valued convex functions defined by:

$$
f_{i}\left(q_{i}\right)=\left\{\begin{array}{lll}
\frac{\left(q_{i}^{-}+\delta_{i}-q_{i}\right)^{2}}{q_{i}-q_{i}^{-}} & \text {if } q_{i} \leqslant q_{i}^{-}+\delta_{i} & \left(0<\delta_{i}<\left(q_{i}^{+}-q_{i}^{-}\right) / 2\right) \\
0 & \text { if } q_{i}^{-}+\delta_{i} \leqslant q_{i} \leqslant q_{i}^{+}-\delta_{i} & \\
\frac{\left(q_{i}^{+}-\delta_{i}-q_{i}\right)^{2}}{q_{i}^{+}-q_{i}} & \text { if } q_{i} \geqslant q_{i}^{+}-\delta_{i}
\end{array}\right.
$$

Note that $h_{\ell}$ is globally convex, and that

$$
h_{\ell}(q)=0 \Longleftrightarrow \frac{\partial h_{\ell}}{\partial q}(q)=0 \Longleftrightarrow q_{i} \in\left[q_{i}^{-}+\delta_{i}, q_{i}^{+}-\delta_{i}\right], \forall i
$$

Finally, $h_{r}$ is a regularization cost function, convex w.r.t. $q$ for any fixed $t$, whose role is to help ensuring the satisfaction of Rule 1 . By choosing

$$
h_{r}(q, t)=\frac{1}{2}(q-\hat{q}(t))^{T} \Gamma(t)(q-\hat{q}(t))
$$

with $\Gamma(t)$ a positive symmetric matrix and $\hat{q}($.$) some function the choice of which is$ discussed a little further, one obtains in view of (18)-(23)

$$
e_{m}=\left(\frac{\partial e_{p}}{\partial q}\right)^{T} e_{p}+\left(\frac{\partial h_{\ell}}{\partial q}\right)^{T}+\Gamma(t)(q-\hat{q}(t))
$$

and

$$
\frac{\partial e_{m}}{\partial q}=\left(\frac{\partial e_{p}}{\partial q}\right)^{T} \frac{\partial e_{p}}{\partial q}+\frac{\partial^{2} h_{\ell}}{\partial q^{2}}+\Gamma(t)+\sum_{k=1}^{n_{q}} e_{p, k} \frac{\partial^{2} e_{p, k}}{\partial q^{2}}
$$

With $h_{\ell}$ defined by (21)-(22), the first two matrices in the right-hand side of (25) are positive. Therefore

$$
\frac{\partial e_{m}}{\partial q} \geqslant \Gamma(t)+\sum_{k=1}^{n_{q}} e_{p, k} \frac{\partial^{2} e_{p, k}}{\partial q^{2}}
$$

Since $e_{p}$ is a smooth function, the invertibility of $\frac{\partial e_{m}}{\partial q}$ on any compact set (Rule 1) can be obtained by choosing $\Gamma(t)$ "positive enough". The choice of $\hat{q}($.$) directly affects$ the satisfaction of Rule 3 . In order to limit the adverse role of $h_{r}$ in this respect, we propose to compute $\hat{q}(t)$ by filtering the measured joint vector $q(t)$ according to

$$
\left\{\begin{aligned}
\dot{\hat{q}} & =-\sigma \frac{\hat{q}-q(t)}{\xi+\|\hat{q}-q(t)\|} \quad(\sigma>0, \xi>0) \\
\hat{q}(0) & =q(0)
\end{aligned}\right.
$$

This ensures that i) $\|\dot{\hat{q}}(t)\|$ is uniformly bounded, ii) $\|\hat{q}(t)-q(t)\|$ is small when $\|\dot{q}(t)\|$ is small itself, and iii) $(\hat{q}(t)-q(t))$, and thus $h_{r}(q(t), t)$, tend to zero when $\dot{q}(t)$ tends to zero. 


\subsection{Guidelines for the choice of the secondary cost-function and the control of the mobile platform}

\subsubsection{Secondary cost-function}

The choice of the secondary cost-function $h_{s}$ will often result from the attempt to satisfy several requirements which may be, in some cases, complementary but also, in other cases, antagonistic. One of them, of particular practical relevance and importance, is to move the mobile platform so that the manipulation task can be carried out without the manipulator's joints colliding into their hardware limits. A cost-function whose minimization ensures this requirement will be denoted as $h_{s, 1}$. At this level of generality, it is hardly possible to specify this function further. Indeed, there may be several ways to fulfill the abovementioned requirement, depending on the properties of the manipulator (its number of d.o.f., for instance, the existence of revolute joints not subjected to limits,...), the nature of the manipulation task, and also the completeness of the information provided by the available sensors about the situation of the mobile platform w.r.t. its environment. Examples of functions $h_{s, 1}$ will however be provided in the next section.

A second typical requirement is to keep the platform motionless whenever this is possible, for instance when the manipulator's joints are "far away" from their limits. The fulfillment of this requirement can be handled via the minimization of a cost-function $h_{s, 2}$ of the form (reminiscent of the regularization function $h_{r}$ evoked previously)

$$
h_{s, 2}\left(\bar{r}_{b}, t\right)=\left(\mathrm{d}_{\gamma}\left(\bar{r}_{b}, \hat{r}(t)\right)\right)^{2}=\left(\mathrm{d}_{\gamma}\left(e, \tilde{r}_{b}\left(\bar{r}_{b}, t\right)\right)\right)^{2}
$$

with $\tilde{r}_{b}\left(\bar{r}_{b}, t\right)=\bar{r}_{b}^{-1} \hat{r}(t)$ computed according to

$$
\begin{aligned}
\dot{\tilde{r}}_{b} & =\bar{R}\left(\tilde{\theta}_{b}\right)\left(\hat{u}(t)-\operatorname{Ad}_{\tilde{r}_{b}^{-1}} \bar{u}_{b}\right) \\
& =\bar{R}\left(\tilde{\theta}_{b}\right) \hat{u}(t)-\left(\begin{array}{cc}
I_{2} & S \tilde{p}_{b} \\
0 & 1
\end{array}\right) \bar{u}_{b} \\
\hat{u}(t) & =-\frac{1}{\xi+d_{\gamma}\left(e, \tilde{r}_{b}(t)\right)} \operatorname{Diag}\left\{\sigma_{i}\right\} \tilde{r}_{b}(t) \quad\left(\sigma_{1,2,3}>0, \xi>0\right) \\
\tilde{r}_{b}(0) & =0
\end{aligned}
$$

The cost $h_{s, 2}$ is minimal when $\tilde{r}_{b}=0$. In view of (29), the satisfaction of this equality on a time-interval implies that $\bar{u}_{b}=0$ on this time-interval. From (13), or (14), this in turn implies that the velocity $u_{b}$ of the mobile platform is equal to zero. This conveys the idea of how the minimization of $h_{s, 2}$ is related to the preoccupation of preventing the mobile platform from moving. However, such a function is not, by itself, very useful, since one can rightfully argue that it suffices to set $u_{b}=0$

$\mathrm{RR} \mathrm{n}^{\circ} 5556$ 
to keep the platform motionless. The real usefulness of this function results from its combination with another cost-function, in order to end-up with a control law containing a term which tends to slow down the mobile platform. For instance, its combination with the control objective represented by the function $h_{s, 1}$ discussed previously yields the following secondary cost-function $h_{s}$ :

$$
h_{s}\left(\bar{r}_{b}, t\right)=\Psi(t) h_{s, 1}\left(\bar{r}_{b}, t\right)+(1-\Psi(t)) h_{s, 2}\left(\bar{r}_{b}, t\right)
$$

with $\Psi(t) \in[0,1]$ denoting a weight function whose derivative is bounded. Examples of such a function will be given further. More generally, $h_{s}$ will be the weighted sum of several terms, each of them associated with a desirable feature concerning the situation of the mobile platform, i.e.

$$
h_{s}\left(\bar{r}_{b}, t\right)=\sum_{i} \Psi_{i}(t) h_{s, i}\left(\bar{r}_{b}, t\right)
$$

\subsubsection{Control of the mobile platform}

Given a secondary cost-function $h_{s}$, the question is then to determine a feedback control $\bar{u}_{b}$ the application of which brings and maintain the value of this function "near" its minimum. In the case where $h_{s}$ does not depend on time and admits a unique minimizing argument $r_{*}$ (as a result of the strict convexity of the function, for instance), a possible control law is

$$
\bar{u}_{b}=-\left(\frac{\partial h_{s}}{\partial \bar{r}_{b}}\left(\bar{r}_{b}\right)\right)^{T} h_{s}\left(\bar{r}_{b}\right)
$$

Indeed, since $\dot{h}_{s}\left(\bar{r}_{b}\right)=\frac{\partial h_{s}}{\partial \bar{r}_{b}}\left(\bar{r}_{b}\right) \bar{u}_{b}$, this control yields in closed loop $\dot{h}_{s}=-\left\|\frac{\partial h_{s}}{\partial \bar{r}_{b}}\left(\bar{r}_{b}\right)\right\|^{2} h_{s}$ $(\leqslant 0)$, which in turn implies that $\left\|\frac{\partial h_{s}}{\partial \bar{r}_{b}}\left(\bar{r}_{b}\right)\right\|^{2} h_{s}\left(\bar{r}_{b}\right)$ tends to zero, and that $h_{s}$ decreases to some limit value $(\geqslant 0)$. If this limit value is not to equal to zero, then $\frac{\partial h_{s}}{\partial \bar{r}_{b}}$ necessarily tends to zero, so that $h_{s}$ tends to its minimum. Therefore, whatever the value of the minimum of $h_{s}$, the above control makes $\bar{r}_{b}$ converge to the minimizing situation $r_{*}$. Note that the calculation of this control does not require the explicit determination of $r_{*}$. The measurement of $h_{s}\left(\bar{r}_{b}\right)$ and its gradient $\frac{\partial h_{s}}{\partial \bar{r}_{b}}\left(\bar{r}_{b}\right)$, plus the property of convexity of the function, are sufficient (just like for any gradient-based minimization algorithm).

The case of a time-dependent function $h_{s}$ is more difficult. In this case, the time derivative of $h_{s}\left(\bar{r}_{b}, t\right)$ along the trajectories of the mobile manipulator is given by

$$
\dot{h}_{s}=a^{T}\left(\bar{r}_{b}, t\right) \bar{u}_{b}+\frac{\partial h_{s}}{\partial t}\left(\bar{r}_{b}, t\right)
$$


with

$$
a^{T}\left(\bar{r}_{b}, t\right)=\frac{\partial h_{s}}{\partial r}\left(\bar{r}_{b}, t\right)
$$

As long as $\frac{\partial h_{s}}{\partial \bar{r}_{b}}\left(\bar{r}_{b}, t\right) \neq 0$, a control value for $\bar{u}_{b}$ which makes $h_{s}$ decrease (i.e. $\dot{h}_{s}<0$ ) can always be found. However, when $\frac{\partial h_{s}}{\partial \bar{r}_{b}}\left(\bar{r}_{b}, t\right)\left(\bar{r}_{b}, t\right)=0$ - which corresponds to a necessary condition for $\bar{r}_{b}$ to be a local minimizer (or maximizer) of $h_{s}$ - the control has no influence on the variation of the function any more. This implies that the minimization of $h_{s}$ at all time-instants cannot be guaranteed in this case. The time evolution of $h_{s}$ will then depend on the choice of the control law, and the properties of $h_{s}$ itself. We propose below an expression for $\bar{u}_{b}$ which, associated with a suitable decomposition of the "drift term" $\frac{\partial h_{s}}{\partial t}$, is "liable" to perform well in many situations.

In what follows, the arguments $\bar{r}_{b}$ and $t$ of all functions will be omitted for the sake of readability.

Consider a decomposition of $\frac{\partial h_{s}}{\partial t}$ in the form

$$
\frac{\partial h_{s}}{\partial t}=a^{T} b_{1}+b_{2}
$$

with $b_{1}$ and $b_{2}$ some functions of $\bar{r}_{b}$ and $t$. Then, in view of (32),

$$
\dot{h}_{s}=a^{T}\left(\bar{u}_{b}+b_{1}\right)+b_{2}
$$

This decomposition is, of course, not unique. It already appears from the above relation that a decomposition with $b_{2}=0$ must be favorable to the minimization of $h_{s}$. However, this choice of $b_{2}$ is not always possible, because $\frac{\partial h_{s}}{\partial t}$ may be different from zero when $\frac{\partial h_{s}}{\partial \bar{r}_{b}}=0$. The relation (35) also shows that $b_{1}$ contains the part of $\frac{\partial h_{s}}{\partial t}$ which can be "exactly" pre-compensated by the control. Let us then consider the problem of minimizing $\frac{1}{2}\left(\bar{u}_{b}+b_{1}\right)^{T} K^{-1}\left(\bar{u}_{b}+b_{1}\right)$ (with $K>0$ ) w.r.t $\bar{u}_{b}$, under the constraint that $\dot{h}_{s}=-\left(a^{T} D a\right)$. The idea, behind this problem, is to i) make $h_{s}$ decrease with a rate (partly) specified by the matrix $D$ (this is the role of the constraint), and ii) among the controls yielding this rate of decrease of $h_{s}$, pick the one whose difference with the pre-compensation of $\frac{\partial h_{s}}{\partial t}$ through the term $b_{1}$ is minimal in the weighted norm associated with the positive matrix $K^{-1}$. This minimization objective is related to energy saving. It also corresponds to the preoccupation of limiting the number of manœuvres during transient phases, when the mobile platform is nonholonomic, by choosing $K$ adequately. More details about this issue can be found in [1]. For instance, by choosing

$$
K(\alpha)=\bar{H}(\alpha) \bar{K} \bar{H}(\alpha)^{T}
$$

$\mathrm{RR} \mathrm{n}^{\circ} 5556$ 
with $\bar{H}(\alpha)$ the matrix defined in (15) and $\bar{K}=\operatorname{Diag}\left\{\bar{k}_{b}, \bar{k}_{b}, \bar{k}_{\alpha}\right\}$ a constant positive matrix, one has $\bar{u}_{b}^{T} K(\alpha)^{-1} \bar{u}_{b}=\left(1 / \bar{k}_{b}\right)\left\|u_{b}\right\|^{2}+\left(1 / \bar{k}_{\alpha}\right) \dot{\alpha}^{2}$. This latter relation in turn suggests to choose $\bar{k}_{b}$ smaller than $\bar{k}_{\alpha}$ in order to penalize the size of platform's velocity $u_{b}$ more than the size of the computed auxiliary control $\dot{\alpha}$. By using (35), the lagrangian associated with the aforementioned minimization problem is

$$
L\left(\bar{u}_{b}\right)=\frac{1}{2}\left(\bar{u}_{b}+b_{1}\right)^{T} K^{-1}\left(\bar{u}_{b}+b_{1}\right)+\lambda\left(a^{T}\left(\bar{u}_{b}+b_{1}\right)+b_{2}+\left(a^{T} D a\right)\right)
$$

with $\lambda$ the Lagrange parameter. The optimality condition

$$
\frac{\partial L}{\partial \bar{u}_{b}}\left(\bar{u}_{b}\right)=0
$$

yields $\bar{u}_{b}=-b_{1}-\lambda K a$, and by using this expression in the constraint equation it follows that

$$
\lambda=\frac{a^{T} D a+b_{2}}{a^{T} K a}
$$

Therefore, the solution to the above minimization problem is

$$
\bar{u}_{b}=-b_{1}-\frac{a^{T} D a}{a^{T} K a} K a-\frac{b_{2}}{a^{T} K a} K a
$$

The regularization of this expression, in order to ensure that $\bar{u}_{b}$ is well defined when $\frac{\partial h_{s}}{\partial \bar{r}_{b}}=0$ (i.e. when $a=0$ ), leads (among other possibilities) to the following control expression

$$
\bar{u}_{b}=-\hat{b}_{1}-\frac{a^{T} D a+\hat{b}_{2}}{a^{T} K a+\mu} K a
$$

with $\mu$ denoting a small positive number, and $\hat{b}_{i}$ (with $i=1,2$ ) some convenient estimation of $b_{i}$ the choice of which is discussed below. From (35), the application of the control law (37) yields, in closed-loop

$$
\dot{h}_{s}=-\frac{a^{T} K a}{a^{T} K a+\mu} a^{T} D a+\frac{\partial h_{s}}{\partial t}-\left(a^{T} \hat{b}_{1}+\frac{a^{T} K a}{a^{T} K a+\mu} \hat{b}_{2}\right)
$$

Let us now comment on the choice of $\left(\hat{b}_{1}, \hat{b}_{2}\right)$ in relation with possible decompositions $\left(b_{1}, b_{2}\right)$ of $\frac{\partial h_{s}}{\partial t}$.

Choice 1: full pre-compensation of $\frac{\partial h_{s}}{\partial t}$ by setting $\left(\hat{b}_{1}=b_{1}, \hat{b}_{2}=b_{2}\right)$.

In this case, the relations (34) and (38) yield

$$
\dot{h}_{s}=-\frac{a^{T} K a}{a^{T} K a+\mu} a^{T} D a+\frac{\mu}{a^{T} K a+\mu} b_{2}
$$


This equation shows that the introduction of the regularizing constant $\mu$ prevents the desired constraint from being satisfied exactly. This was expected. It also points out the fact that the pre-compensation of $\frac{\partial h_{s}}{\partial t}$ is all the more efficient, in terms of the minimization of $h_{s}$, that $\left\|b_{2}\right\|$ is small. Indeed, when $b_{2} \equiv 0$, then $h_{s}$ is non-increasing, and $a$, and thus $\frac{\partial h_{s}}{\partial \bar{r}_{b}}$, converge to zero. If $h_{s}$ is strictly convex with respect to the variable $\bar{r}_{b}$ and does not depend on time, this in turn implies that $h_{s}$ converges to its minimum. When $b_{2} \neq 0$, it is no longer possible to ensure that $h_{s}$ converges to a minimum. Nevertheless, the following relation (which is a direct consequence of $(39))$

$$
\left(a^{T} D a\right)\left(a^{T} K a\right) \geqslant \mu b_{2} \Longrightarrow \dot{h}_{s} \leqslant 0
$$

indicates that the decrease of $h_{s}$ is all the more likely that $\left\|b_{2}\right\|$ is small. When $b_{2}$ cannot be taken equal to zero, a possible simple decomposition $\left(b_{1}, b_{2}\right)$ of $\frac{\partial h_{s}}{\partial t}$, coherent with the rule of keeping $\left\|b_{2}\right\|$ small, is, for instance

$$
b_{1}=\frac{\partial h_{s}}{\partial t} \frac{1}{\|a\|^{2}+\nu} a \Rightarrow b_{2}=\frac{\partial h_{s}}{\partial t} \frac{\nu}{\|a\|^{2}+\nu}
$$

with $\nu$ denoting a small positive number.

Choice 2: no pre-compensation of $\frac{\partial h_{s}}{\partial t}$ by setting $\left(\hat{b}_{1}=0, \hat{b}_{2}=0\right)$.

Common consequences of this choice are a less "reactive" control and a degradation of performance, in terms of minimizing $h_{s}$. On the other hand, a beneficial counterpart of this loss of reactivity is a reduction of the control effort. This effect can be foreseen from the constrained optimization problem considered for the control design, after remarking that setting $b_{1}=0$ yields the minimization of $\bar{u}_{b}^{T} K^{-1} \bar{u}_{b}$. In view of (38), the variation of $h_{s}$ is then given by

$$
\dot{h}_{s}=-\frac{a^{T} K a}{a^{T} K a+\mu} a^{T} D a+\frac{\partial h_{s}}{\partial t}
$$

Choice 3: pre-compensation of $\frac{\partial h_{s}}{\partial t}$ combined with a reduction of the control effort, by setting $\left(\hat{b}_{1}=0, \hat{b}_{2}=\frac{\partial h_{s}}{\partial t}\right)$.

This choice corresponds to the decomposition $\left(b_{1}=0, b_{2}=\frac{\partial h_{s}}{\partial t}\right)$ of $\frac{\partial h_{s}}{\partial t}$. In this case, the control effort is reduced (for the same reasons as in the previous case) at the expense of the pre-compensation of $\frac{\partial h_{s}}{\partial t}$, which is not achieved exactly, and, subsequently, at the expense of the minimization of $h_{s}$. This control solution can be seen as intermediary between Choices 1 and 2. Obviously, there is also a continuum of possibilities involving partial pre-compensation of $\frac{\partial h_{s}}{\partial t}$, each of them achieving a different compromise in terms of control energy expenditure and minimization of $h_{s}$.

$\mathrm{RR} \mathrm{n}^{\circ} 5556$ 
From there, a deeper analysis of the control properties requires to specify the function $h_{s}$ further, as illustrated by an example treated in the next section.

Let us finally recall that the control $u_{b}$ of the physical mobile platform is calculated from $\bar{u}_{b}$ by using the relation (13) (or (14)), i.e. $u_{b}=\bar{u}_{b}$, when the mobile platform is omnidirectional, and $\left(v_{b, 1}, \omega_{b}, \dot{\alpha}\right)^{T}=\bar{H}(\alpha)^{-1} \bar{u}_{b}$ with the matrix $\bar{H}(\alpha)$ defined in (15), when the platform is nonholonomic and unicycle-like. Let us also shortly comment on the existence of limitations upon the velocity components of the platform, i.e. upon the components of $u_{b}$. A simple way to handle these limitations consists in "saturating" the control. In this case, it is useful to have in mind that the saturation should be implemented by dividing all the components of $\left(u_{b}, \dot{\alpha}\right)$ by the same number (greater or equal to one), so as to augment the chances of preserving the stabilization properties of the control.

\section{Application examples}

\subsection{Target tracking in $\mathrm{SE}(2)$}

This application, depicted on Fig. 2, consists in tracking in both position and orientation an object (materialized on the figure by the frame $\mathcal{F}_{t}$ ) moving in the plane. The mobile manipulator consists of a unicycle-like platform equipped with a threejoint RPR manipulator. The axis of the first joint is attached to the platform at the point of coordinates $(L, 0)$ w.r.t. the frame $\mathcal{F}_{b}$. The distance between the last joint's axis and the origin $E$ of the end-effector's frame $\mathcal{F}_{e}$ is denoted by $L_{e}$.

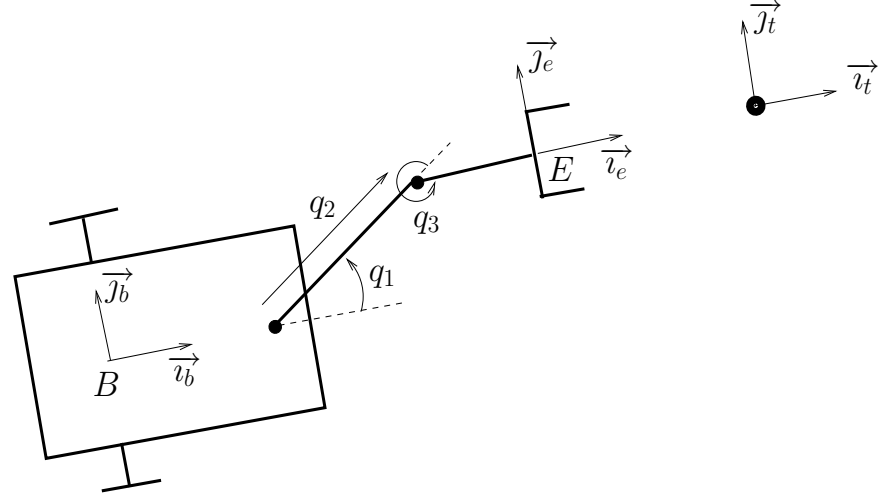

Figure 2: Tracking in $\mathrm{SE}(2)$ 


\subsubsection{The manipulation task and associated control}

The manipulation objective is the stabilization of the target's situation w.r.t. the end-effector, $r_{e t}$, at a fixed desired value $r_{e t}^{*}=\left(X^{*}, 0,0\right)^{T}$. The realization of this objective implies that, whatever the target's motion in the robot's environment, the target appears to be motionless with respect to the end-effector, at a distance $X^{*}$ from it. This is a three-dimensional virtual linkage. One can assume, for instance, that $r_{e t}$ is measured via the observation of the target by a camera mounted on the end-effector. With the notation of Section 3.2, a possible choice for the task-function $e_{p}$ associated with this manipulation objective is

$$
e_{p}\left(q, r_{b}, t\right)=\tilde{r}_{e t}:=\left(r_{e t}^{*}\right)^{-1} r_{e t}
$$

In order to calculate the joint control (17), one needs to determine the partial derivatives of $e_{p}$ w.r.t. $q, r_{b}$, and $t$. Since $\theta_{e t}^{*}=0$ and $u_{e t}^{*}=0$, one deduces from (8) and (42) that

$$
\dot{e}_{p}=\dot{r}_{e t}
$$

Since $r_{e t}=r_{e b} r_{b t}$, one also has, by using (4) and (9),

$$
\dot{r}_{e t}=\bar{R}\left(\theta_{e t}\right)\left(u_{b t}+\operatorname{Ad}_{r_{t b}} u_{e b}\right)
$$

with

$$
u_{b t}=u_{t}-\operatorname{Ad}_{r_{t b}} u_{b}
$$

Therefore

$$
\dot{r}_{e t}=\bar{R}\left(\theta_{e t}\right)\left(u_{t}+\operatorname{Ad}_{r_{t b}}\left(u_{e b}-u_{b}\right)\right)
$$

Now, $r_{e b}$ (the situation of the mobile platform w.r.t. the manipulator's end-effector) is a function of $q$ only, and it is in fact simple to verify that

$$
r_{e b}=\left(\begin{array}{c}
-R\left(-q_{1}-q_{3}\right)\left(\begin{array}{c}
L \\
0
\end{array}\right)-R\left(-q_{3}\right)\left(\begin{array}{c}
q_{2} \\
0
\end{array}\right)-\left(\begin{array}{c}
L_{e} \\
0
\end{array}\right) \\
-q_{1}-q_{3}
\end{array}\right)
$$

Therefore

$$
\begin{aligned}
\dot{r}_{e b} & =\bar{R}\left(\theta_{e b}\right) u_{e b} \\
& =\frac{\partial r_{e b}}{\partial q} \dot{q}
\end{aligned}
$$

$\mathrm{RR} \mathrm{n}^{\circ} 5556$ 
and, from (43)-(44),

$$
\dot{e}_{p}=\bar{R}\left(\theta_{e t}\right)\left(u_{t}+\operatorname{Ad}_{r_{t b}}\left(\bar{R}\left(\theta_{b e}\right) \frac{\partial r_{e b}}{\partial q} \dot{q}-u_{b}\right)\right)
$$

With the definition specified by (10) of the partial derivatives of a function, one deduces from the above equation (by a term-by-term identification) that

$$
\begin{aligned}
& \frac{\partial e_{p}}{\partial q}=\bar{R}\left(\theta_{e t}\right) \operatorname{Ad}_{r_{t b}} \bar{R}\left(\theta_{b e}\right) \frac{\partial r_{e b}}{\partial q}=\left(\begin{array}{cc}
I_{2} & R\left(\theta_{e b}\right) S p_{b t} \\
0 & 1
\end{array}\right) \frac{\partial r_{e b}}{\partial q} \\
& \frac{\partial e_{p}}{\partial r_{b}}=-\bar{R}\left(\theta_{e t}\right) \operatorname{Ad}_{r_{t b}}=-\left(\begin{array}{cc}
R\left(\theta_{e b}\right) & R\left(\theta_{e b}\right) S p_{b t} \\
0 & 1
\end{array}\right) \\
& \frac{\partial e_{p}}{\partial t}=\bar{R}\left(\theta_{e t}\right) u_{t}
\end{aligned}
$$

with $\theta_{e b}=-\theta_{b e}=-\left(q_{1}+q_{3}\right)$. One easily verifies from (45) that $\frac{\partial r_{e b}}{\partial q}$, and thus $\frac{\partial e_{p}}{\partial q}$, are invertible everywhere except at $q_{2}=0$.

Having determined $e_{p}, e_{m}$ is given by (18)-(19), with $h_{p}$ given by (20), $h_{l}$ by (21) $-(22)$, and $h_{r}$ by (23) and (27). The control $u_{q}$ is then given by (17). The various (constant) parameters involved in these expressions will be specified further.

\subsubsection{Secondary cost-function and control of the mobile platform}

For this application, if the mobile platform were omnidirectional, an obvious way to ensure the avoidance of the joint's limits while tracking the target would consist in keeping the relative situation $r_{t b}=r_{t}^{-1} r_{b}$ between the platform and the target equal to some nominal (constant) value $r_{*}$. Since the mobile platform is subjected to nonholonomic contraints, this objective cannot be achieved whatever the target's motion. However, one can try to "nearly" obtain this result by controlling the platform's companion frame instead. This suggests to consider the following secondary cost-function $h_{s, 1}$

$$
h_{s, 1}\left(\bar{r}_{b}, t\right)=\frac{1}{2}\left(\mathrm{~d}_{\gamma}\left(r_{*},\left(r_{t}(t)\right)^{-1} \bar{r}_{b}\right)\right)^{2}=\frac{1}{2}\left(\mathrm{~d}_{\gamma}(e, \tilde{r})\right)^{2}
$$

with $\mathrm{d}_{\gamma}$ denoting the distance defined by (2), and $\tilde{r}=r_{*}^{-1} r_{t}^{-1} \bar{r}_{b}$. One deduces from (8) and (12) that

$$
\dot{\tilde{r}}=\bar{R}(\tilde{\theta})\left(\bar{u}_{b}-\operatorname{Ad}_{\left(r_{*} \tilde{r}\right)^{-1}} u_{t}\right)
$$

with $u_{t}$ defined by $\dot{r}_{t}=\bar{R}\left(\theta_{t}\right) u_{t}$. From (2),

$$
\frac{\partial\left(\mathrm{d}_{\gamma}(e, \tilde{r})\right)^{2}}{\partial \tilde{r}}=2(\tilde{x}, \tilde{y}, \gamma \sin \tilde{\theta}) \bar{R}(\tilde{\theta})
$$


so that, by (50), Eq. (32) is satisfied for the function $h_{s}=h_{s, 1}$ with

$$
\begin{aligned}
& a^{T}\left(\bar{r}_{b}, t\right)=(\tilde{x}, \tilde{y}, \gamma \sin \tilde{\theta}) \bar{R}(\tilde{\theta}) \\
& \frac{\partial h_{s}}{\partial t}\left(\bar{r}_{b}, t\right)=(\tilde{x}, \tilde{y}, \gamma \sin \tilde{\theta}) \bar{R}(\tilde{\theta})\left(-\operatorname{Ad}_{\left.\left(r_{*} \tilde{r}\right)^{-1} u_{t}\right)}\right.
\end{aligned}
$$

These equations also indicate that, in this case, a decomposition of $\frac{\partial h_{s}}{\partial t}$ in the form $\frac{\partial h_{s}}{\partial t}=a^{T} b_{1}+b_{2}$ is obtained by setting

$$
b_{1}\left(\bar{r}_{b}, t\right)=-\operatorname{Ad}_{\left(r_{*} \tilde{r}\right)^{-1}} u_{t}, \quad b_{2}=0
$$

The application of the control (37) with $\hat{b}_{1}=b_{1}$ and $\hat{b}_{2}=b_{2}$ then makes $h_{s}$ decrease (in view of (39) when $b_{2}=0$ ) and, in fact, makes this cost converge to zero. In this case, it is not difficult to show that, by choosing the parameters $\varepsilon_{1}$ and $\varepsilon_{2}$ of the transverse function small enough, and provided that i) the initial situation of the end-effector w.r.t. the target is not too far from its ideal value, ii) the initial situation $r_{t b}$ of the mobile platform w.r.t. the target is not too far from $r^{*}$, and iii) the matrix $\Gamma$ involved in the regularization cost $h_{r}(q, t)$ is chosen equal to zero, then exponential stabilization to zero of the tracking error (between the target and the end-effector) is obtained, with the manipulator's joints staying away from their limits. This result holds whatever the motion of the target, provided that its velocity $u_{t}$ is measured (or estimated) precisely.

Now, the realization of the secondary task, as specified above, implies that the mobile platform has to move whenever the target moves. If one would prefer to keep the platform motionless until one of the manipulator's joints get close to one of its limits (before the exact execution of the manipulation task has to be abandoned in order to avoid a collision with the joint limit), one should consider a combination of $h_{s, 1}$ with another cost function, like the function $h_{s, 2}$ specified by relations (28) and (29). This yields the secondary cost-function of relation (30), i.e.

$$
h_{s}\left(\bar{r}_{b}, t\right)=\Psi(t) h_{s, 1}\left(\bar{r}_{b}, t\right)+(1-\Psi(t)) h_{s, 2}\left(\bar{r}_{b}, t\right)
$$

with $\Psi$ denoting a weight function the determination of which is now discussed. The idea is to have $\Psi(t)$ equal to zero as long as the joints coordinates $q$ are far from their limits, and to have $\Psi(t)$ strictly positive otherwise. Let us however recall that $\Psi$ cannot be a function of $q$ (since, otherwise, $h_{s}$ would no longer be a function of $\bar{r}_{b}$ and $t$ only). It has to be a function of time whose derivative is bounded. In order to comply with this constraint, a possibility consists in filtering $\tilde{\Psi}(q(t))$, with $q(t)$ the measured joint vector, and $\tilde{\Psi}$ a function whose value is equal to zero when the joint

$\mathrm{RR} \mathrm{n}^{\circ} 5556$ 
coordinates are far from their limits, and strictly positive otherwise. An example of such a function is

$$
\tilde{\Psi}(q)=\frac{1}{n_{q}} \sum_{i=1}^{n_{q}} \tilde{\sigma}_{i}^{2}\left(q_{i}\right), \quad \tilde{\sigma}_{i}\left(q_{i}\right)=\left\{\begin{array}{cl}
1 & \text { if } q_{i} \leqslant q_{i}^{-}+\delta_{i}^{--} \text {or } q_{i} \geqslant q_{i}^{+}-\delta_{i}^{++} \\
\frac{q_{i}^{-}+\delta_{i}^{-}-q_{i}}{\delta_{i}^{-}-\delta_{i}^{--}} & \text {if } q_{i}^{-}+\delta_{i}^{--}<q_{i}<q_{i}^{-}+\delta_{i}^{-} \\
0 & \text { if } q_{i}^{-}+\delta_{i}^{-} \leqslant q_{i} \leqslant q_{i}^{+}-\delta_{i}^{+} \\
\frac{q_{i}-q_{i}^{+}-\delta_{i}^{+}}{\delta_{i}^{+}-\delta_{i}^{++}} & \text {if } q_{i}^{+}-\delta_{i}^{+}<q_{i}<q_{i}^{+}-\delta_{i}^{++}
\end{array}\right.
$$

with the margins $\delta_{i}^{+}$and $\delta_{i}^{-}$chosen larger than the margins $\delta_{i}$ used in (21), in order to reduce the risk for the manipulator arm to reach a joint configuration beyond which the perfect execution of the manipulation task is abandoned due to that $h_{l}(q) \neq 0$. The margins $\delta_{i}^{++}$and $\delta_{i}^{--}$are then chosen inside the intervals $\left(\delta_{i}, \delta_{i}^{+}\right)$and $\left(\delta_{i}, \delta_{i}^{-}\right)$ respectively.

A possible filtering of $\tilde{\Psi}(q(t))$ is as follows (compare with (27)):

$$
\left\{\begin{aligned}
\dot{\Psi} & =-\lambda \frac{\Psi-\tilde{\Psi}(q(t))}{\xi+\|\Psi-\tilde{\Psi}(q(t))\|} \quad(\lambda>0, \xi>0) \\
\Psi(0) & =\tilde{\Psi}(q(0))
\end{aligned}\right.
$$

Let us remark that $\Psi(t) \in[0,1]$ for any $t$ (because $\tilde{\Psi}(q) \in[0,1]$ for any $q$ ), so that $\Psi$ corresponds to a weight function. Note also that the larger $\lambda$, the weaker the filtering.

A slightly different possibility for the determination of $\Psi(t)$ consists in setting

$$
\Psi(t)=\frac{1}{n_{q}} \sum_{i=1}^{n_{q}} \sigma_{i}^{2}(t)
$$

with $\sigma_{i}(t)$ denoting a filtered value of $\tilde{\sigma}_{i}\left(q_{i}(t)\right)$ numerically calculated as follows:

$$
\left\{\begin{aligned}
\dot{\sigma}_{i} & =-\lambda \frac{\sigma_{i}-\tilde{\sigma}_{i}\left(q_{i}(t)\right)}{\xi+\sigma_{i}-\sigma_{i}\left(q_{i}(t) \mid\right.} \quad(\lambda>0, \xi>0) \\
\sigma_{i}(0) & =\tilde{\sigma}_{i}\left(q_{0}\right)
\end{aligned}\right.
$$

A third possibility, which reduces the computational load, consists in setting

$$
\Psi(t)=\frac{1}{n_{q}} \sum_{i=1}^{n_{q}} \sigma_{i}^{2}\left(\hat{q}_{i}(t)\right)
$$

with $\hat{q}_{i}\left(i=1, \ldots, n_{q}\right)$ the components of the filtered function $\hat{q}$ of $q$ defined by (27). 


\subsubsection{Simulation results}

The geometric parameters of the mobile manipulator have been chosen as follows:

- $L=0.4 \mathrm{~m}, L_{e}=0.15 \mathrm{~m}$,

- $\left(q_{1}^{-}, q_{1}^{+}\right)=\left(-\frac{\pi}{3}, \frac{\pi}{3}\right) \mathrm{rad},\left(q_{2}^{-}, q_{2}^{+}\right)=(0,0.7) \mathrm{m}$, and $\left(q_{3}^{-}, q_{3}^{+}\right)=\left(-\frac{\pi}{2}, \frac{\pi}{2}\right) \mathrm{rad}$.

The manipulation task-function $e_{m}$ is designed as described in Section 3.2, with the function $e_{p}$ given by (42) with $X^{*}=0.5(\mathrm{~m})$. The parameters involved in the definition of $h_{\ell}$ (Eq. (21) and (22)) are $\alpha_{i}=1, \delta_{i}=0.05\left(q_{i}^{+}-q_{i}^{-}\right)$. The regularization term $h_{r}$ is given by $(23)$, with $\Gamma(t)=0.2 \exp (-t) I_{3}$. The gain matrix in the definition of $u_{q}$ (Eq. (17)) is $G=3 I_{3}$.

Concerning the calculation of the mobile platform control, the function $h_{s}$ is given by (30), with $h_{s, 1}$ and $h_{s, 2}$ given by (49) and (28)-(29) respectively. The parameters used in the definition of $h_{s, 1}$ are $\gamma=1$ (for the distance $\mathrm{d}_{\gamma}$ ), and $r_{*}=$ $-\left(X^{*}+L_{e}+L+0.45,0,0\right)^{T}$. This value has been chosen so that $e_{p}=0$ when $r_{t b}=r_{*}$ and $q=q_{*}=(0,0.45,0)^{T} \in \prod_{i}\left(q_{i}^{-}, q_{i}^{+}\right)$. The parameters used in the definition of $h_{s, 2}$ are $\gamma=1, \sigma_{i}=10$, and $\xi=1$. Two choices of $\Psi$ (specified below) have been considered. The control $\bar{u}_{b}$ is then calculated according to (37) with $K$ given by (36) for $\bar{K}=\operatorname{Diag}(1,1,100), D=I_{3}$, and $\mu=10^{-4}$. The functions $\hat{b}_{1}$ and $\hat{b}_{2}$ will be specified below. The transverse function $f$, from which $H(\alpha)$ and $\bar{H}(\alpha)$ can be calculated, is defined by $(16)$ with $\left(\varepsilon_{1}, \varepsilon_{2}\right)=(0.2,0.4)$. The control $u_{b}$ for the mobile platform is then calculated from (14). There remains to specify the functions $\Psi$, $\hat{b}_{1}$, and $\hat{b}_{2}$. In order to illustrate the influence of these functions on the closed-loop system's behavior, we consider four possible choices below and present simulation results for two different trajectories of the target.

Target's trajectory 1: For the simulation results reported on Fig. 3-6, the amplitude of the target's motion is kept small so that, once the platform has come close enough to the target, the manipulation objective can be achieved with the mobile platform at rest. The target's motion is defined by the velocity $u_{t}$ (i.e. $\dot{r}_{t}=\bar{R}\left(\theta_{t}\right) u_{t}$ ) with

$$
u_{t}(t)=-\frac{\pi}{20}\left(\sin \frac{\pi}{2} t, \cos \frac{\pi}{2} t, 0\right)^{T}
$$

This corresponds to a circular motion of the origin of the target's frame, with a fixed orientation of this frame (see Fig. 3 where the target's frame and the associated circular motion are represented on the upper-right part of the figures). The initial situation of the target w.r.t. the platform is $r_{b t}(0)=(2.6,1,0)^{T}$, and the initial 
configuration of the manipulator arm is $q(0)=(0,0.35,0)^{T}$ (with $q$ as specified on Fig. 2). We now detail the different choices made for $\Psi, \hat{b}_{1}$, and $\hat{b}_{2}$.

(a) $\Psi=1$, and $\left(\hat{b}_{1}, \hat{b}_{2}\right)$ is set according to Choice $\mathbf{1}$ in Section 3.3 .2 , i.e. $\left(\hat{b}_{1}, \hat{b}_{2}\right)=$ $\left(b_{1}, b_{2}\right)$ with $b_{1}, b_{2}$ defined by $(51)$. Since $\Psi=1, h_{s}$ is equal to $h_{s, 1}$. Then, due to the definition of $\left(\hat{b}_{1}, \hat{b}_{2}\right)$, and since $b_{2}=0$, the convergence of $h_{s}$ to zero is guaranteed in this case, as this can be observed on Fig. 6(a). The cartesian motion of the mobile manipulator is shown on Fig. 3(a). One can distinguish two phases. During the first part of the simulation, the platform approaches the target and both $h_{s}$ and $e_{p}$ tend to zero (Fig. 6(a) and 4(a)). This transient convergence phase is followed by a steady behavior which involves periodic (non-stationary) motions of both the platform and manipulator. Although $h_{s}$ and $e_{p}$ are very well controlled to zero, a practical drawback of this solution is that it does not allow the mobile platform to remain still. This fact is also illustrated by Fig. 5(a) which shows how the control inputs $v_{b, 1}$ and $\omega_{b}$ evolve with time.

(b) $\Psi(t)$ is defined by (53), and $\left(\hat{b}_{1}, \hat{b}_{2}\right)$ is again set according to Choice $\mathbf{1}$ in Section 3.3.2, except that $b_{1}$ and $b_{2}$ are now defined by (40) with $\nu=10^{-4}$. The parameters $(\lambda, \xi)=(1.66,1), \delta_{i}^{-}=0.2\left(q_{i, *}-q_{i}^{-}\right), \delta_{i}^{+}=0.2\left(q_{i}^{+}-q_{i, *}\right)$, $\delta_{i}^{--}=\frac{2}{3} \delta_{i}^{-}$, and $\delta_{i}^{++}=\frac{2}{3} \delta_{i}^{+}$have been used in the definition of $\tilde{\Psi}$ (Eq. (52)). The main difference w.r.t. the previous case concerns the definition of $h_{s}$, and the effect of inertia resulting from the function $h_{s, 2}$. Fig. 3(b) and 5(b) confirm that this strategy reduces the amplitude of the platform's displacements. This does not impede the achievement of the manipulation objective, as shown by Fig. 4(b). Note that although $h_{s}$ does no longer tend to zero, due to that $b_{2} \neq 0$, it is ultimately small (Fig. $6(\mathrm{~b})$ ).

(c) $\Psi(t)$ is defined as above (Simulation $(\mathrm{b})$ ), and $\left(\hat{b}_{1}, \hat{b}_{2}\right)$ is set according to Choice 2 in Section 3.3.2, i.e. $\left(\hat{b}_{1}, \hat{b}_{2}\right)=(0,0)$. By comparison with the previous simulation, this choice yields a much smoother motion of the platform and convergence to a fixed posture. The manipulation objective is again perfectly achieved after the transient phase is extinct, although this phase lasts longer (Fig 4(c)).

(d) $\Psi(t)$ is defined as above, and $\left(\hat{b}_{1}, \hat{b}_{2}\right)$ is set according to Choice 3 in Section 3.3.2, i.e. $\left(\hat{b}_{1}, \hat{b}_{2}\right)=\left(0, \frac{\partial h_{s}}{\partial t}\right)$. This corresponds to a compromise between the choices made in Simulations (b) and (c), since it allows to partially precompensate the drift term $\frac{\partial h_{s}}{\partial t}$ in (38). For this type of target's motion, the 
resulting behavior of the mobile manipulator is not much different from the one obtained in Simulation (c).

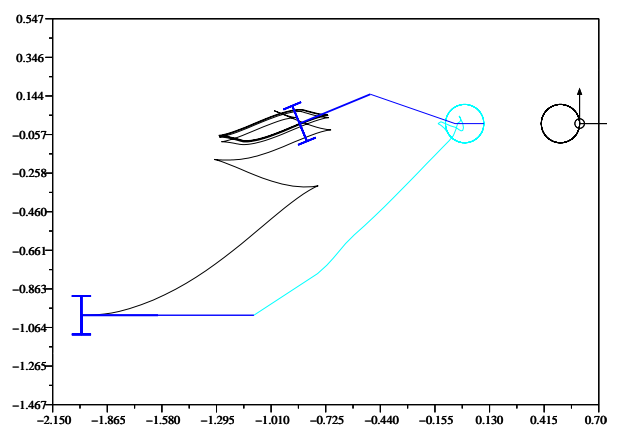

(a)

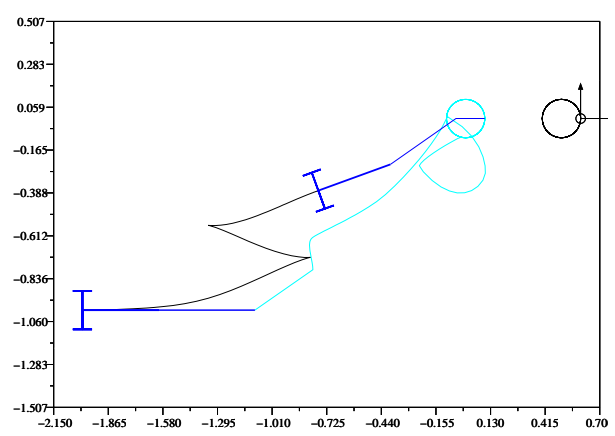

(c)

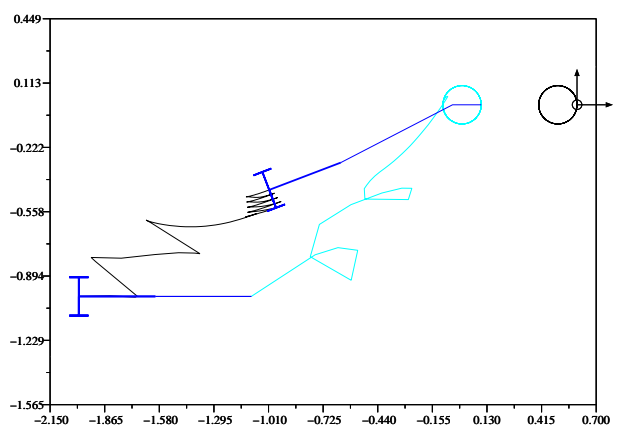

(b)

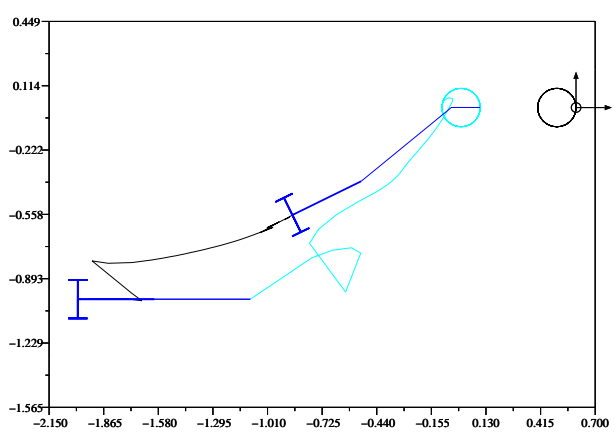

(d)

Figure 3: Target's trajectory 1: cartesian trajectories of the mobile manipulator and target.

$\mathrm{RR} \mathrm{n}^{\circ} 5556$ 


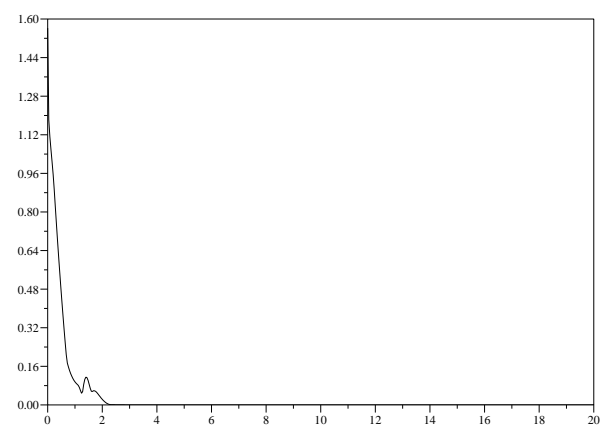

(a)

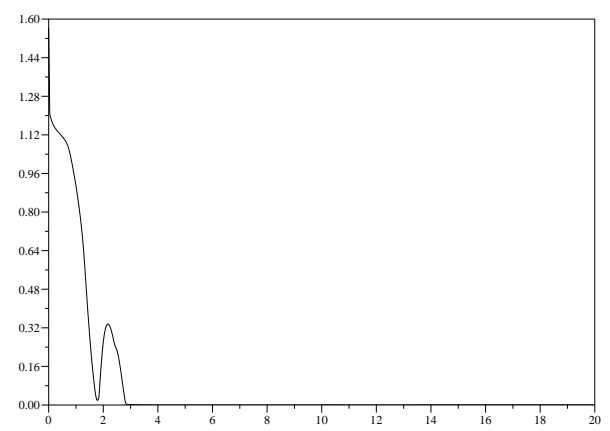

(c)

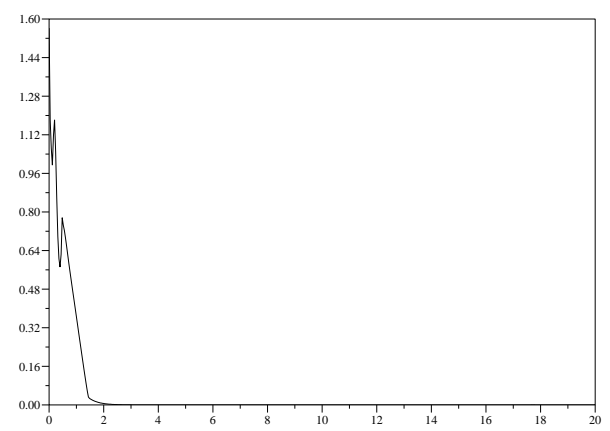

(b)

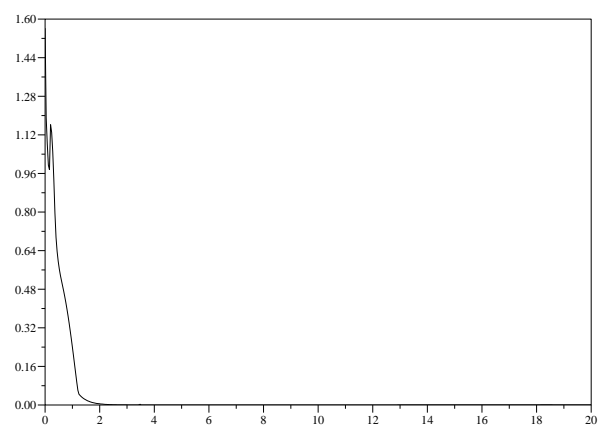

(d)

Figure 4: Target's trajectory 1: norm of the function $e_{p}$ associated with the manipulation objective. 


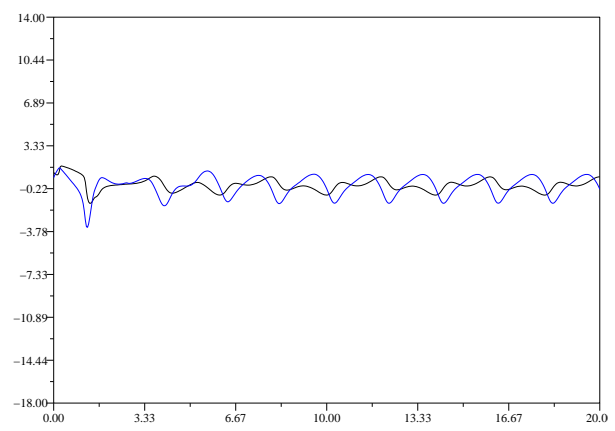

(a)

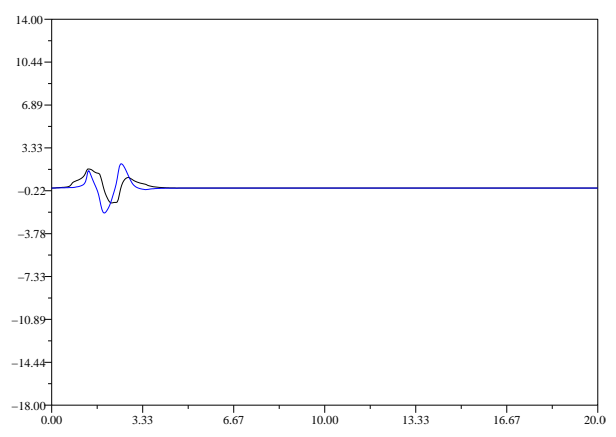

(c)

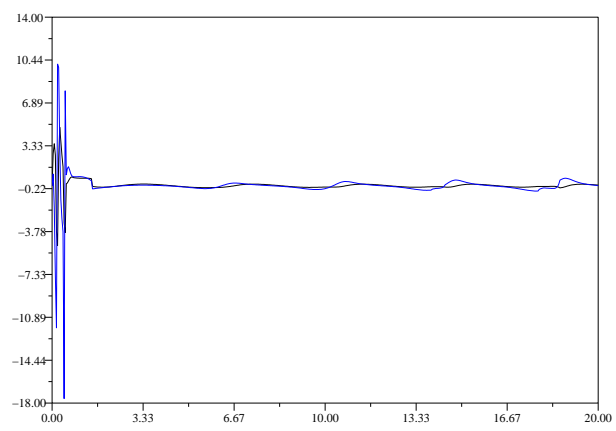

(b)

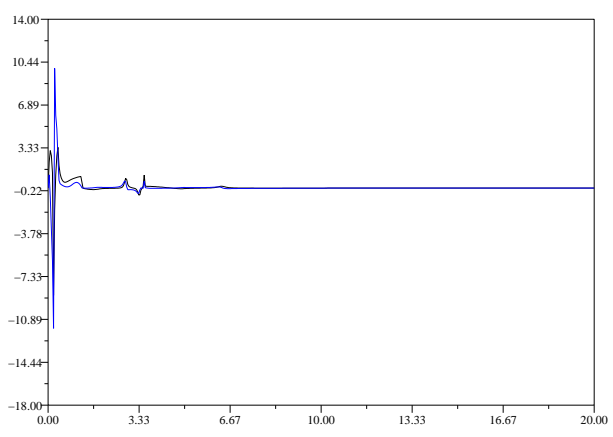

(d)

Figure 5: Target's trajectory 1: mobile platform velocities $v_{b, 1}$ and $\omega_{b}$.

$\mathrm{RR} \mathrm{n}^{\circ} 5556$ 


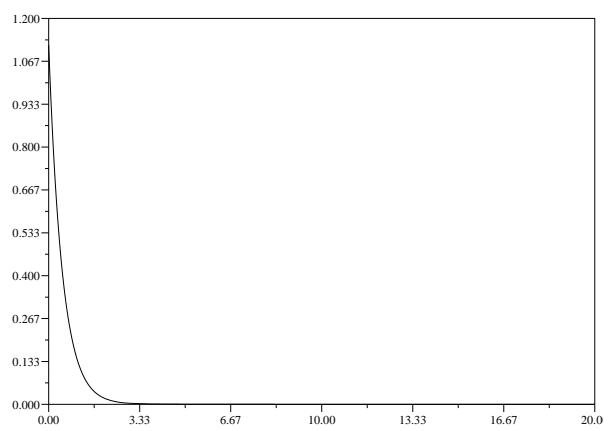

(a)

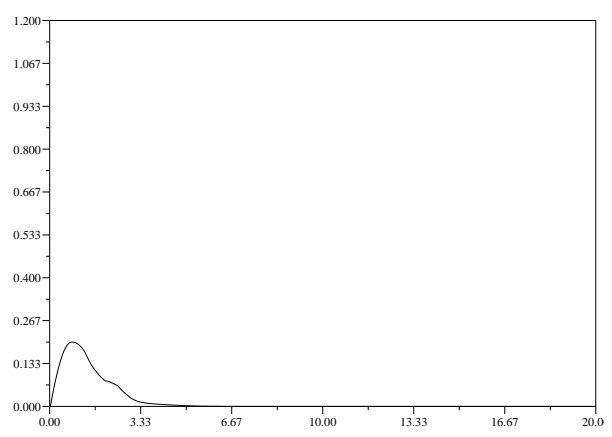

(c)

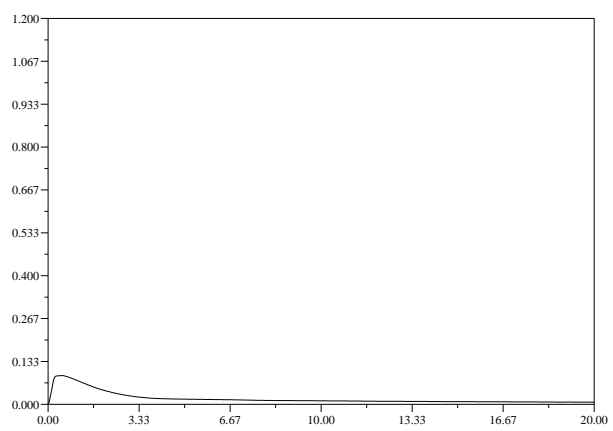

(b)

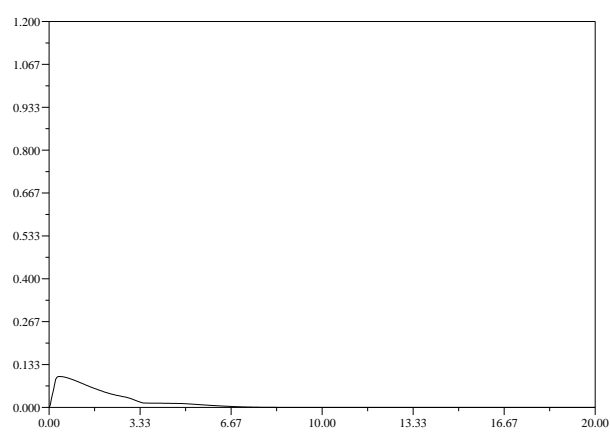

(d)

Figure 6: Target's trajectory 1: secondary cost-function $h_{s}$. 
Target's trajectory 2: For the simulation results reported on Fig. 7-10, the same control parameters as above have been considered and tested with the four different choices of $\Psi, \hat{b}_{1}$, and $\hat{b}_{2}$ corresponding to Simulations (a), (b), (c), and (d). However, the target's motion is now more complex and its larger amplitude is such that, during some time intervals, the platform has to move and execute manœuvres in order to allow for the tracking of the target by the manipulator's end-effector. The initial situation of the target w.r.t. the platform is $r_{b t}(0)=(2.5,1,0)^{T}$, and the initial configuration of the manipulator arm is $q(0)=(0,0.35,0)^{T}$. The target's velocity $u_{t}$ is specified in Table 1.

Table 1: Target's velocity for trajectory 2

\begin{tabular}{ll}
\hline Time interval in $s$ & Target's velocity $\left(\right.$ in m.s $\left.\mathrm{s}^{-1},{\mathrm{~m} . \mathrm{s}^{-1} \text { and rad.s }}^{-1}\right)$ \\
\hline$t \in[0,5)$ & $u_{t}(t)=(0,0,0)^{T}$ \\
$t \in[5,27)$ & $u_{t}(t)=\left(0,0, \frac{\pi^{2}}{20} \cos \frac{\pi(t-5)}{2}\right)^{T}$ \\
$t \in[27,37)$ & $u_{t}(t)=(0.2,0,0)^{T}$ \\
$t \in[37,59)$ & $u_{t}(t)=\left(0,0, \frac{\pi^{2}}{20} \cos \frac{\pi(t-37)}{2}\right)^{T}$ \\
$t \in[59,99)$ & $u_{t}(t)=(0,0.05,0)^{T}$ \\
$t \in[99,105)$ & $u_{t}(t)=(0,0,0)^{T}$ \\
$t \in[105,115)$ & $u_{t}(t)=(-0.2,0,0)^{T}$ \\
$t \in[115,120)$ & $u_{t}(t)=(0,0,0)^{T}$ \\
\hline
\end{tabular}

From this table, and as one can observe on Fig. 7, the trajectory of the origin of the target's frame is composed of three straight lines. Contrary to the previous set of simulations, the orientation of the target is no longer constant. The simulation results (Fig. 7-10) basically confirm the observations made in the previous case. In particular,

1. The choice (a), for the control design, yields an excellent tracking of the target by the manipulator's end-effector, but this performance is obtained at the expense of the steady motion of the mobile platform and numerous manœuvres.

2. The choice (b) reduces the number of the platform's manœuvres, but the platform's velocities are still large during some of these manœuvres. Besides, this control strategy does no longer ensure the perfect tracking of the target by the manipulator's end-effector all the time. The problem occurs at times when the delayed manœuvering of the mobile platform, in order to catch-up with the evading target, prevents the manipulation objective from being achieved without a manipulator's joint colliding into one of its limits. 
3. By comparison with Choice (b), the choices (c) and (d) yield even fewer manœuvres and smaller platform's velocities. The amount of time during which the tracking of the target by the manipulator's end-effector is perfectly executed is not much different, eventhough the frequency of the time intervals during which perfect tracking is lost does not seem to be favorable to the choice (b) (see Fig. 8(b), 8(c), and 8(d)). In this respect, and as expected from the analysis, the choice (d) produces a slightly more reactive and effective control than the choice (c). This is coherent with sharper variations of the platform velocities, as this can be observed from Fig. 9(c) and 9(d). 


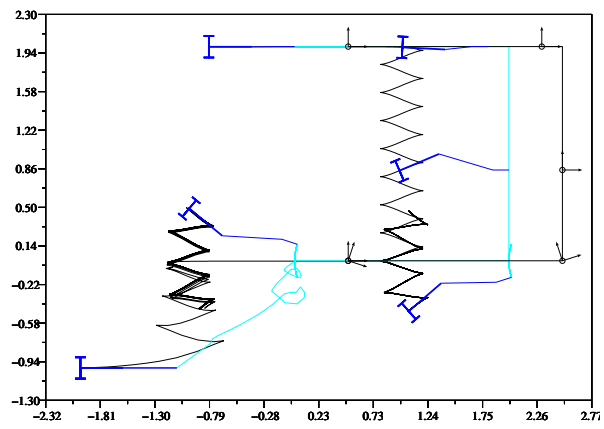

(a)

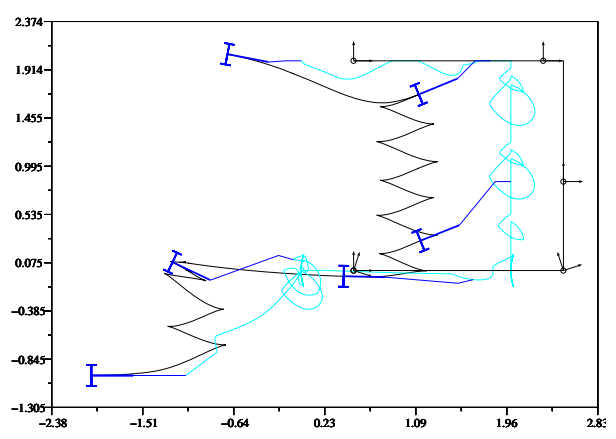

(c)

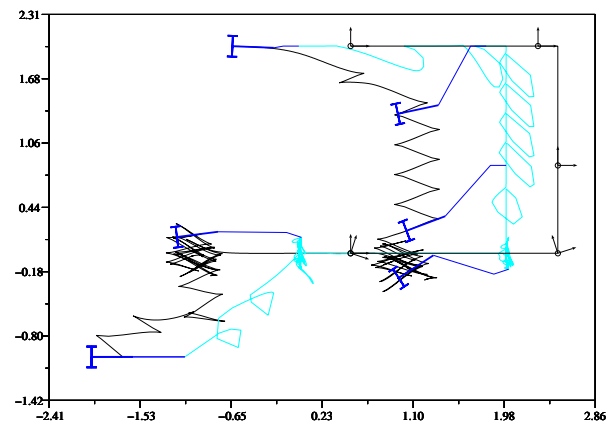

(b)

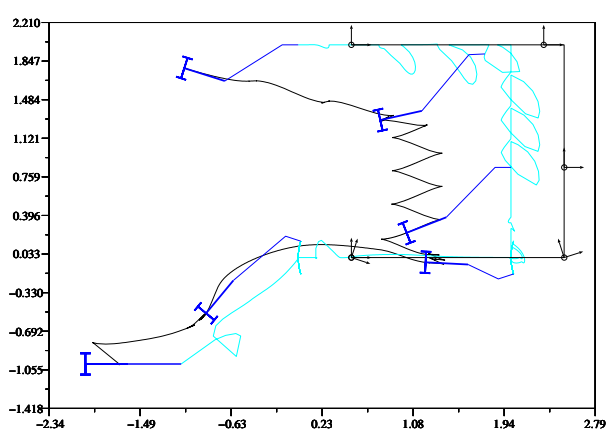

(d)

Figure 7: Target's trajectory 2: cartesian trajectories of the mobile manipulator and target.

$\mathrm{RR} \mathrm{n}^{\circ} 5556$ 


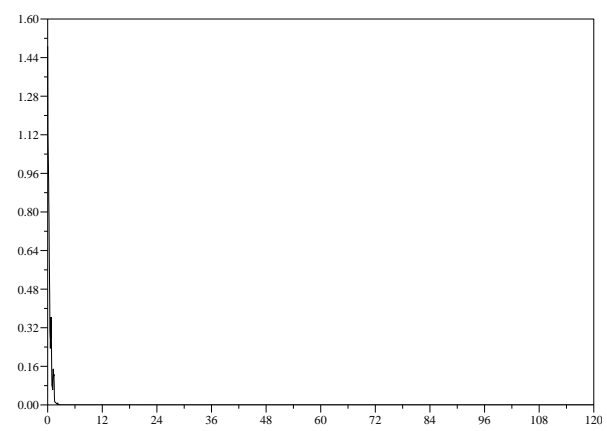

(a)

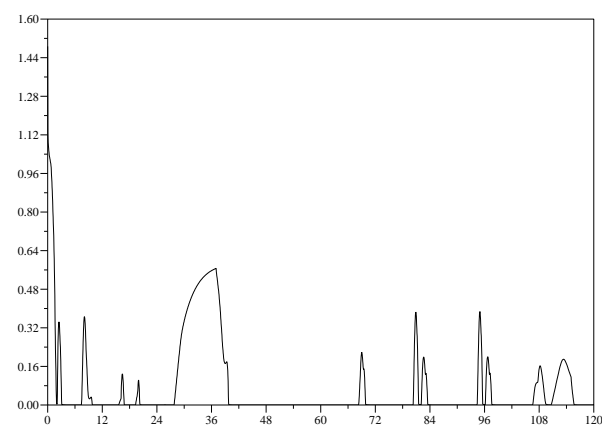

(c)

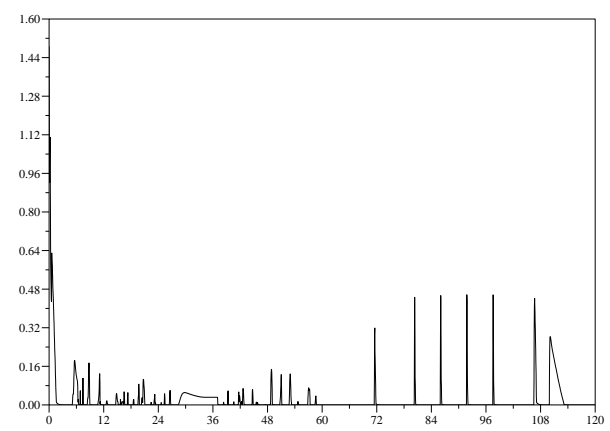

(b)

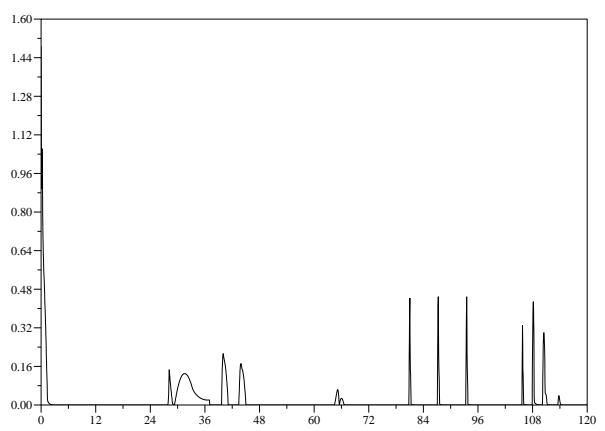

(d)

Figure 8: Target's trajectory 2: norm of the function $e_{p}$ associated with the manipulation objective. 


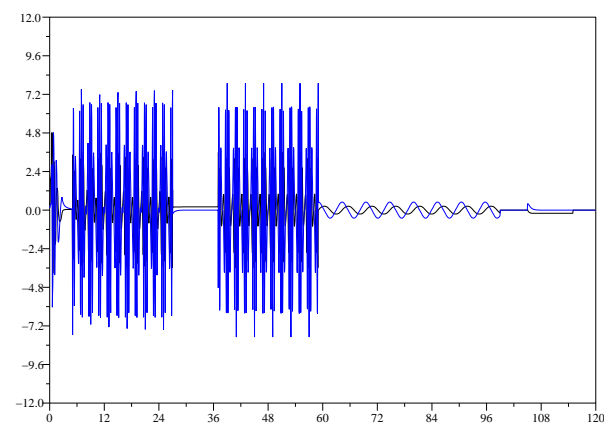

(a)

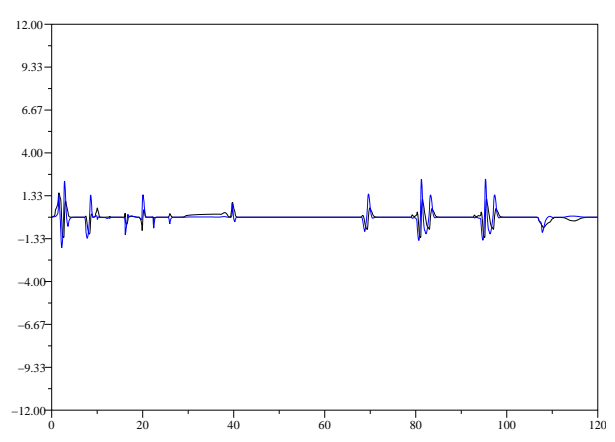

(c)

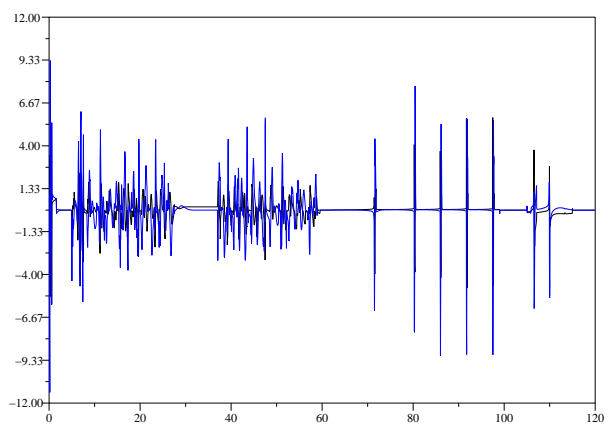

(b)

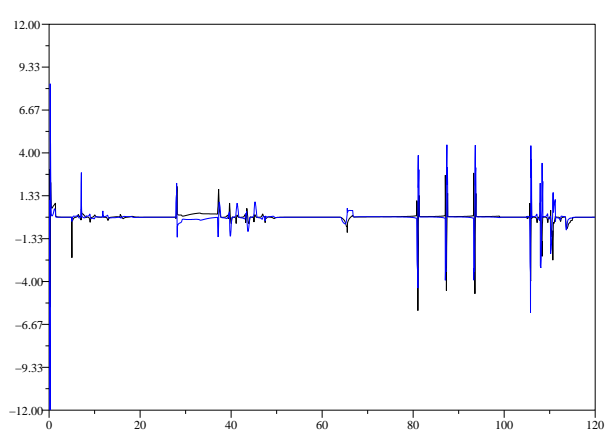

(d)

Figure 9: Target's trajectory 2: mobile platform velocities $v_{b, 1}$ and $\omega_{b}$.

$\mathrm{RR} \mathrm{n}^{\circ} 5556$ 


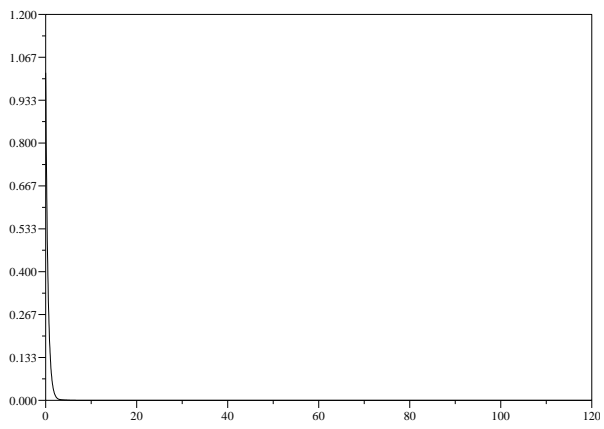

(a)

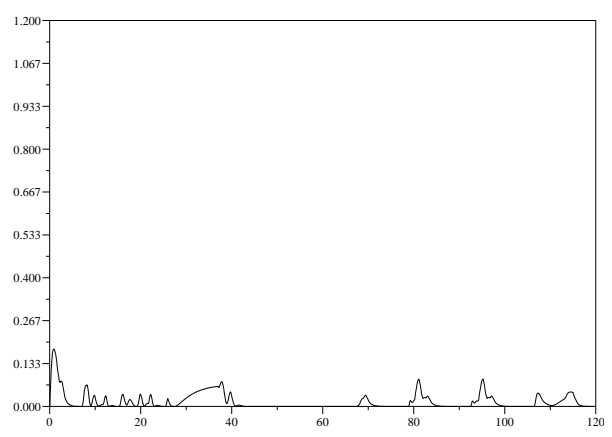

(c)

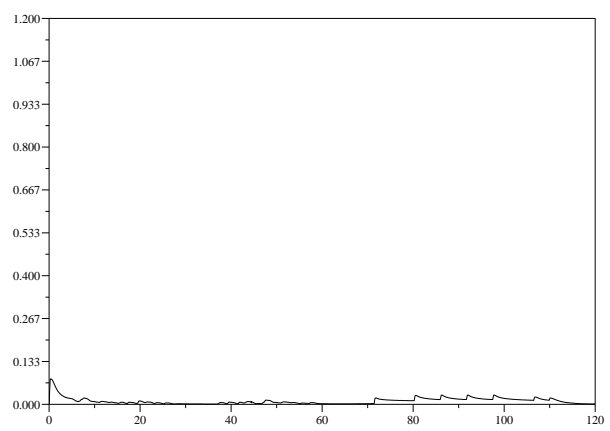

(b)

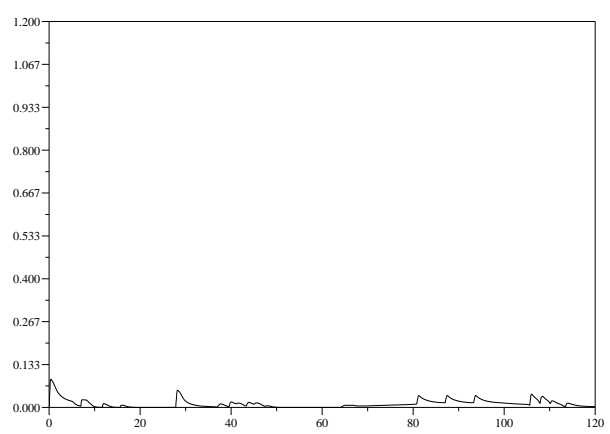

(d)

Figure 10: Target's trajectory 2: secondary cost-function $h_{s}$. 


\subsection{A tracking application in $\mathbb{R}^{2}$}

We now consider the problem of tracking in position an object materialized on Fig. 11 by the point $T$ with a mobile manipulator composed of a unicycle-like platform and a two-joints RP manipulator. The first link of the manipulator is attached to the platform by a revolute joint at the point of coordinates $(L, 0)$ w.r.t. the frame $\mathcal{F}_{b}$. The control design closely follows the procedure used for the previous example.

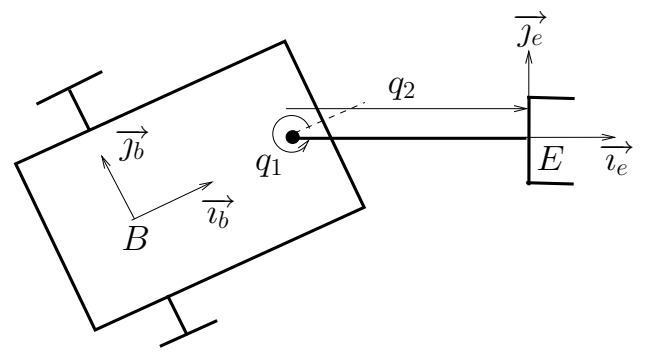

Figure 11: Tracking in $\mathbb{R}^{2}$

\subsubsection{Manipulation task and associated control}

The manipulation objective is a tracking task consisting in having the manipulator's end-effector point toward $T$ while keeping the distance between the origin $E$ of the end-effector and $T$ equal to some desired value $X^{*}$. This is equivalent to the stabilization of $p_{e t}$, the target's position w.r.t. the end-effector's frame $\mathcal{F}_{e}$ (expressed in $\mathcal{F}_{e}$ ), at the reference value $p_{e t}^{*}=\left(X^{*}, 0\right)^{T}$. This suggests, among other possibilities, to define $e_{p}$ as

$$
e_{p}\left(q, r_{b}, t\right)=\tilde{p}_{e t}:=p_{e t}-p_{e t}^{*}
$$

The partial derivatives of $e_{p}$ can then be obtained either by a direct calculation, or by using the fact that $\tilde{p}_{e t}$ is the position component of $\tilde{r}_{e t}$ defined by (42) (recall that $\left.\theta_{e t}^{*}=0\right)$. From $(48)$, one deduces that

$$
\begin{aligned}
& \frac{\partial e_{p}}{\partial q}=\left(\begin{array}{ll}
I_{2} & R\left(\theta_{e b}\right) S p_{b t}
\end{array}\right) \frac{\partial r_{e b}}{\partial q} \\
& \frac{\partial e_{p}}{\partial r_{b}}=-\left(\begin{array}{ll}
R\left(\theta_{e b}\right) & R\left(\theta_{e b}\right) S p_{b t}
\end{array}\right) \\
& \frac{\partial e_{p}}{\partial t}=R\left(-\theta_{e}\right) \dot{p}_{t}
\end{aligned}
$$


From the relations

$$
r_{e b}=\left(\begin{array}{c}
\left.-R\left(-q_{1}\right)\left(\begin{array}{c}
L \\
0
\end{array}\right)-\left(\begin{array}{c}
q_{2} \\
0
\end{array}\right)\right) \\
-q_{1}
\end{array}\right)
$$

and $\theta_{e b}=-\theta_{b e}=-q_{1}$, one can verify that $\frac{\partial e_{p}}{\partial q}$ is invertible everywhere, except when $q_{2}+x_{e t}=0$

From here, the manipulation task $e_{m}$ and the control $u_{q}$ for the manipulator arm are defined as indicated in Subsection 3.2. The parameters involved in these definitions will be specified further.

\subsubsection{Secondary cost function and control of the platform}

For this application, one can specify a vector $p_{*}$ such that, when $p_{b t}$ belongs to a neighborhood of $p_{*}$, the manipulation objective can be perfectly achieved (i.e. $e_{p}$ can be maintained equal to zero) via the control of $q$ inside the allowed domain $\left(q_{1}^{-}, q_{1}^{+}\right) \times\left(q_{2}^{-}, q_{2}^{+}\right)$. Since $p_{b t}$ is the position component of $r_{b t}=\left(r_{b}\right)^{-1} r_{t}$, this suggests to define $h_{s, 1}$ by

$$
h_{s, 1}\left(\bar{r}_{b}, t\right)=\frac{1}{2}\left\|\tilde{p}-p_{*}\right\|^{2}
$$

with $\tilde{p}$ the position component of $\bar{r}_{b}^{-1} r_{t}=f(\alpha) r_{b}^{-1} r_{t}$. Let us remark that

$$
\tilde{p}=p_{f}+R\left(\theta_{f}\right) p_{b t}=p_{f}+R\left(\theta_{f}-\theta_{b}\right)\left(p_{t}-p_{b}\right)
$$

can be calculated from the knowledge of the transverse function $f$ and from the relative position $p_{b t}$ of the target w.r.t. the platform. By using (5) and (8), one shows that

$$
\dot{\tilde{p}}=R\left(-\bar{\theta}_{b}\right) \dot{p}_{t}-\left(I_{2} \quad S \tilde{p}\right) \bar{u}_{b}
$$

Therefore, $\dot{h}_{s, 1}=a^{T} \bar{u}_{b}+\frac{\partial h_{s, 1}}{\partial t}$ (i.e. Eq. (32)) with

$$
\begin{array}{ll}
a^{T}\left(\bar{r}_{b}, t\right) & =-\left(\tilde{p}-p_{*}\right)^{T}\left(I_{2} \quad S \tilde{p}\right) \\
\frac{\partial h_{s, 1}}{\partial t} & =\left(\tilde{p}-p_{*}\right)^{T} R\left(-\bar{\theta}_{b}\right) \dot{p}_{t}
\end{array}
$$

and a possible decomposition of $\frac{\partial h_{s, 1}}{\partial t}$ in the form $\frac{\partial h_{s, 1}}{\partial t}=a^{T} b_{1}+b_{2}$ is given by

$$
b_{1}\left(\bar{r}_{b}, t\right)=-\left(\begin{array}{c}
R\left(-\bar{\theta}_{b}\right) \dot{p}_{t} \\
0
\end{array}\right), \quad b_{2}\left(\bar{r}_{b}, t\right)=0
$$


As in the previous application, one can define the secondary cost function $h_{s}$ as a weighted sum (30) of $h_{s, 1}$ and the function $h_{s, 2}$ defined by (28). The expressions for the weight function $\Psi$ given in Section 4.1 .2 can also be used for the present application.

\subsubsection{Simulation results}

For the simulations results presented below, the geometric parameters of the mobile parameters have been chosen as follows:

- $L=0.4 \mathrm{~m}$,

- $\left(q_{1}^{-}, q_{1}^{+}\right)=\left(-\frac{\pi}{3}, \frac{\pi}{3}\right) \mathrm{rad}$, and $\left(q_{2}^{-}, q_{2}^{+}\right)=(0.15,0.85) \mathrm{m}$,

The manipulation task $e_{m}$ is designed as described in Section 3.2, with the function $e_{p}$ given by $(55)$ for $X^{*}=0.5(\mathrm{~m})$. The parameters used in the definition of $h_{\ell}$ (Eq. (21) and (22)) are $\alpha_{i}=1$ and $\delta_{i}=0.05\left(q_{i}^{+}-q_{i}^{-}\right)$, and the regularization cost $h_{r}$ is given by $(23)$ with $\Gamma(t)=0.6 \exp (-t) I_{2}$. Finally, the gain matrix in the definition of $u_{q}$ (Eq. (17)) is $G=3 I_{2}$.

Concerning the calculation of the mobile platform control, the function $h_{s}$ is given by (30), with $h_{s, 1}$ and $h_{s, 2}$ given by (57) and (28)-(29) respectively. The reference vector $p_{*}=\left(X^{*}+L+0.5,0\right)^{T}$ has been chosen so that the satisfaction of $h_{s, 1}=0$ implies that $e_{p}=0$ is obtained for $q_{*}=(0,0.5)^{T}$. The parameters used in the definition of $h_{s, 2}$ are $\gamma=1, \sigma_{i}=10$, and $\xi=1$. The control $\bar{u}_{b}$ is defined by (37) with $K$ given by (36) for $\bar{K}=\operatorname{Diag}(1,1,100), D=I_{3}$, and $\mu=10^{-4}$. The functions $\hat{b}_{1}$ and $\hat{b}_{2}$ will be specified below. The transverse function $f$ is defined by (16) with $\left(\varepsilon_{1}, \varepsilon_{2}\right)=(0.25,0.6)$. The control $u_{b}$ for the mobile platform is then calculated from $(14)$.

The initial position of the target with respect to the platform is $p_{b t}=(2,-1.7)^{T}$, and the initial configuration of the manipulator arm is $q(0)=(0,0.5)^{T}$. The target's velocity $\dot{p}_{t}$ is specified in Table 2 .

There remains to specify the functions $\Psi, \hat{b}_{1}$, and $\hat{b}_{2}$. Four choices have been considered, corresponding to the four strategies already tested for the tracking application of Section 4.1. The simulation results reported on Fig. 12-15 confirm the conclusions drawn from the tracking application of Section 4.1. In addition, they indicate that, for the present application, the choice (d) seems to achieve the best compromise in terms of target tracking performance (Fig. 13) and smoothness of the platform's motion with few manœuvres and small velocities (Fig. 12 and 14).

$\mathrm{RR} \mathrm{n}^{\circ} 5556$ 


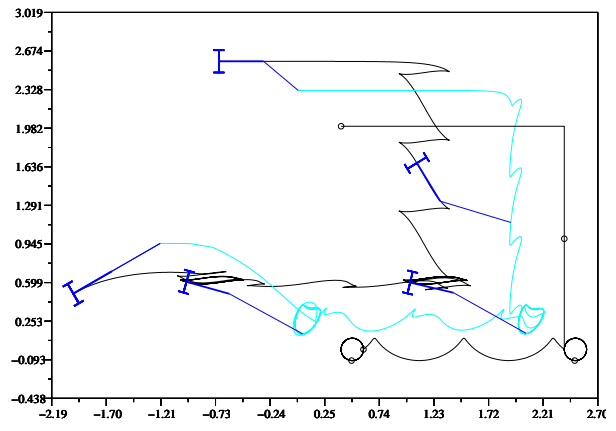

(a)

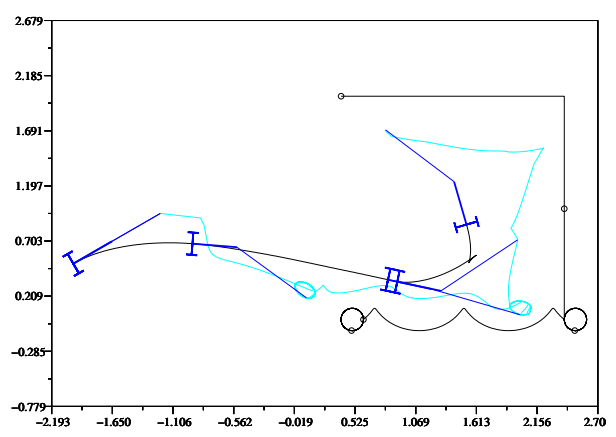

(c)

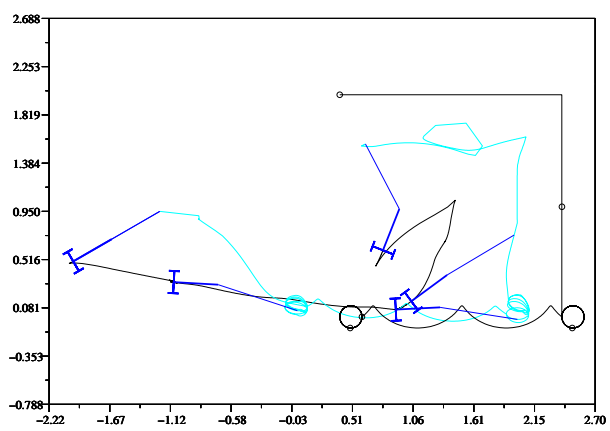

(b)

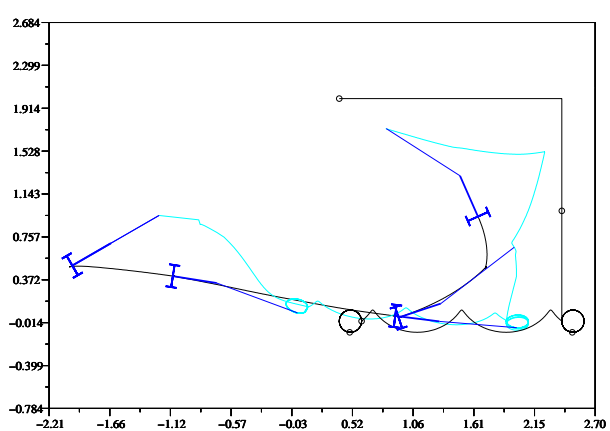

(d)

Figure 12: Cartesian trajectories of the mobile manipulator and target. 


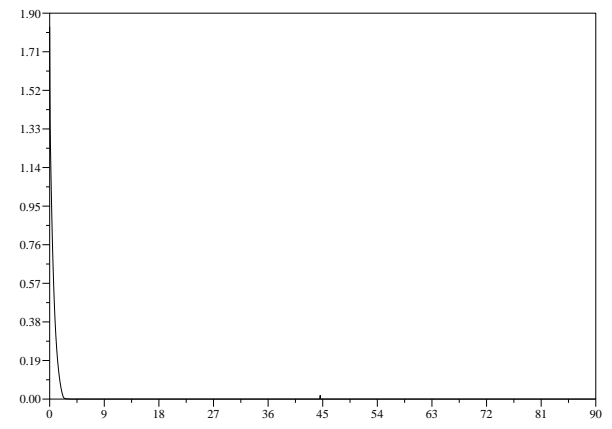

(a)

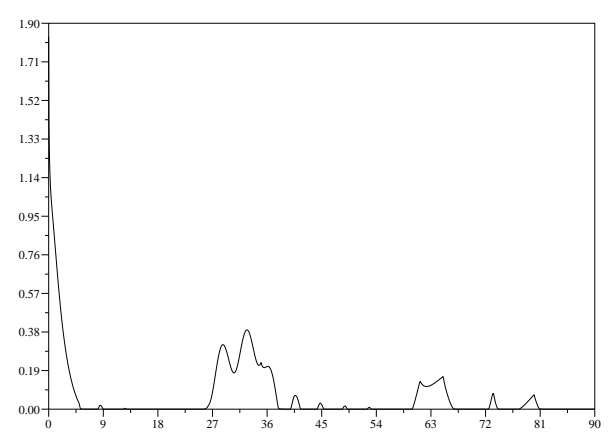

(c)

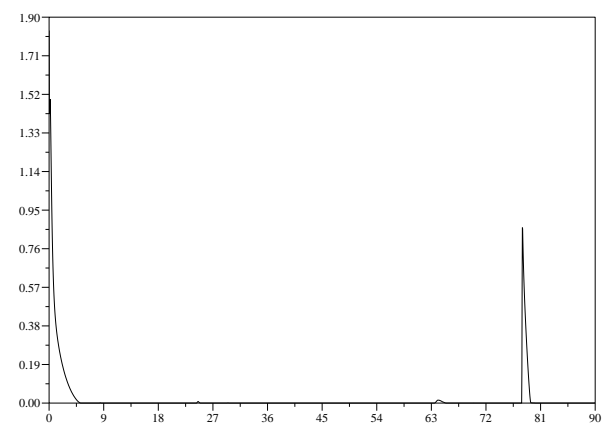

(b)

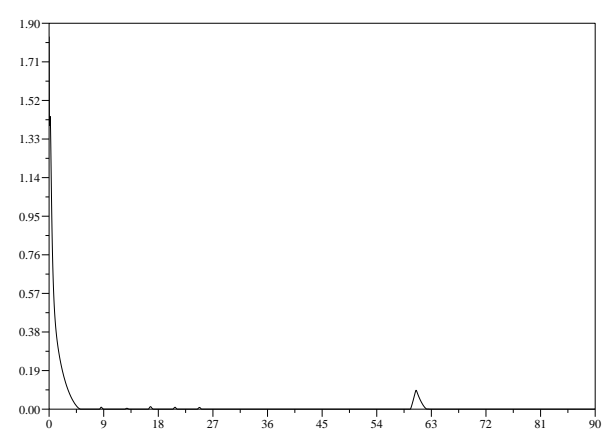

(d)

Figure 13: Norm of the function $e_{p}$ associated with the manipulation objective.

$\mathrm{RR} \mathrm{n}^{\circ} 5556$ 


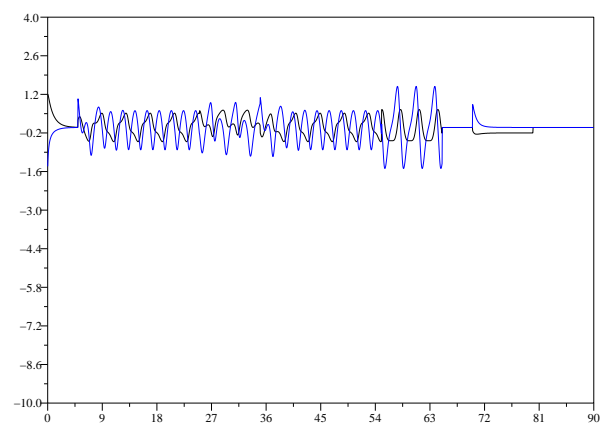

(a)

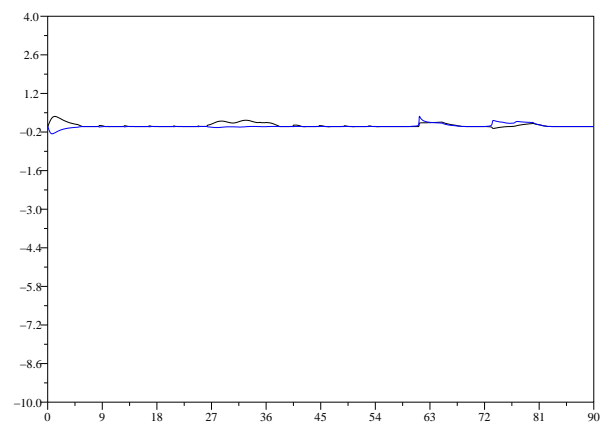

(c)

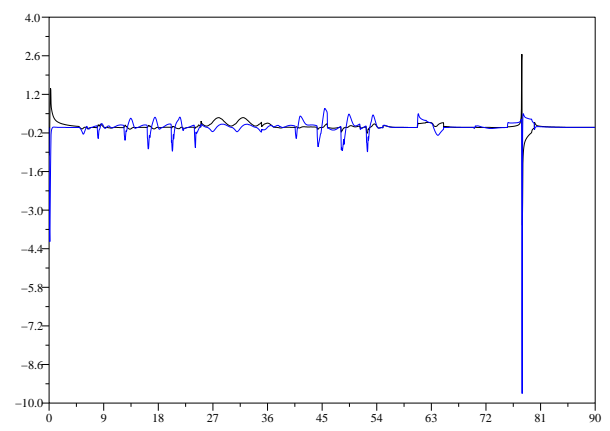

(b)

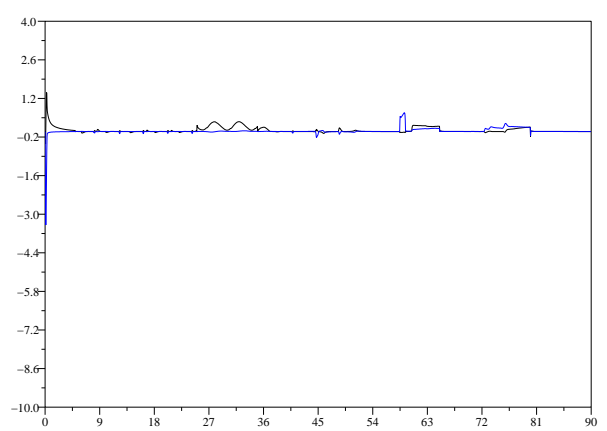

(d)

Figure 14: Mobile platform velocities $v_{b, 1}$ and $\omega_{b}$.

INRIA 


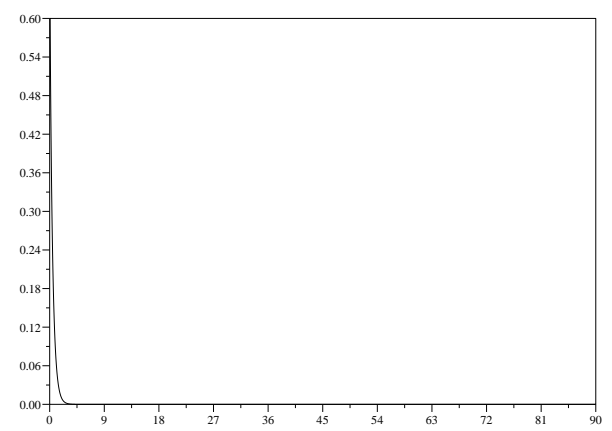

(a)

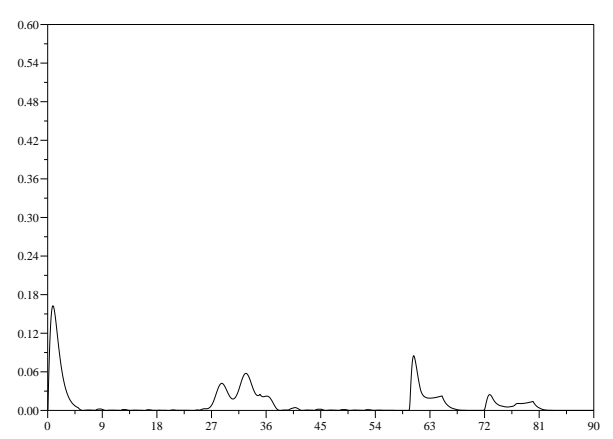

(c)

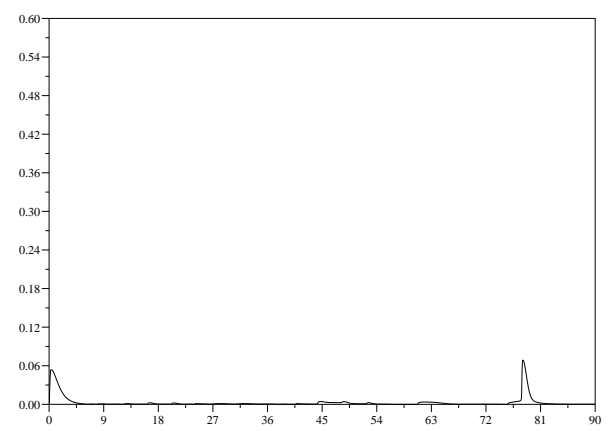

(b)

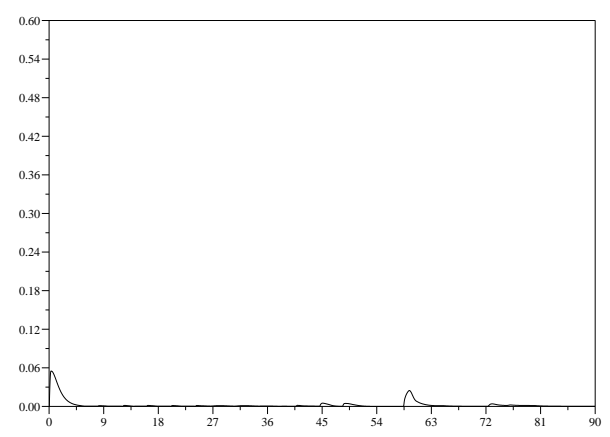

(d)

Figure 15: Secondary cost-function $h_{s}$.

$\mathrm{RR} \mathrm{n}^{\circ} 5556$ 
Table 2: Target's velocity $\dot{p}_{t}$

\begin{tabular}{ll}
\hline Time interval in $s$ & Target's velocity $\left(\right.$ in m.s $\left.s^{-1}\right)$ \\
\hline$t \in[0,5)$ & $\dot{p_{t}}=(0,0)^{T}$ \\
$t \in[5,25)$ & $\dot{p_{t}}=\left(-\frac{\pi}{20} \sin \frac{\pi(t-5)}{2}, \frac{\pi}{20} \cos \frac{\pi(t-5)}{2}\right)^{T}$ \\
$t \in[25,35)$ & $\dot{p_{t}}=\left(0.2-\frac{\pi}{20} \sin \frac{\pi(t-5)}{2}, \frac{\pi}{20} \cos \frac{\pi(t-5)}{2}\right)^{T}$ \\
$t \in[35,55)$ & $\dot{p_{t}}=\left(-\frac{\pi}{20} \sin \frac{\pi(t-5)}{2}, \frac{\pi}{20} \cos \frac{\pi(t-5)}{2}\right)^{T}$ \\
$t \in[55,65)$ & $\dot{p_{t}}=(0,0.2)^{T}$ \\
$t \in[65,70)$ & $\dot{p_{t}}=(0,0)^{T}$ \\
$t \in[70,80)$ & $\dot{p_{t}}=(-0.2,0)^{T}$ \\
$t \in[80,90]$ & $\dot{p_{t}}=(0,0)^{T}$ \\
\hline
\end{tabular}

\section{Extension to more general mobile manipulators}

Some extensions of the present approach, beyond the framework of planar mobile manipulators with a unicycle-like platform, are now discussed. First of all, it is important to remark that the approach applies directly to general manipulators. For example, tracking problems in $S E(3)$ with conventional six degrees-of-freedom manipulator arms can be addressed in the same way as in Section 4 for applications in $S E(2)$. Then, the approach can also be extended to other types of mobile platforms. Unicycle-like platforms are specific because their kinematic equations can be described by a left-invariant system on the Lie group $S E(2)$. Although the transverse function approach is best suited for systems on Lie groups, it is not restricted to this framework. For example, the case of car-like platforms (and, more generally, the case of $N$-trailers systems) can also be addressed via minor modifications of the control design presented in Section 3. Let us show this.

The control design methodology described in Section 3 relies on the fact that $\bar{r}_{b}$ is the configuration of an omnidirectional companion frame $\mathcal{F}_{\bar{b}}(\alpha)$, close to $\mathcal{F}_{b}$. In the case of a unicycle-like platform, we have shown in Section 2.3 how $\bar{r}_{b}$ can be defined from the transverse function approach. We explain next how the results of Section 2.3 (and, therefore, the whole control approach) can be extended to a car-like platform. 
With the notation of Section 2, the kinematic equations of a car-like platform w.r.t. the fixed frame $\mathcal{F}_{0}$ are given by

$$
\left\{\begin{array}{l}
\dot{x}_{b}=v_{b, 1} \cos \theta_{b} \\
\dot{y}_{b}=v_{b, 1} \sin \theta_{b} \\
\dot{\theta}_{b}=v_{b, 1} \xi \\
\dot{\xi}=v_{\xi}
\end{array}\right.
$$

with $\xi=(\tan \varphi) / \ell, \varphi$ the steering angle of the vehicle (see Fig. 16 below), and $\ell$ the distance between the front and rear wheels' axles.

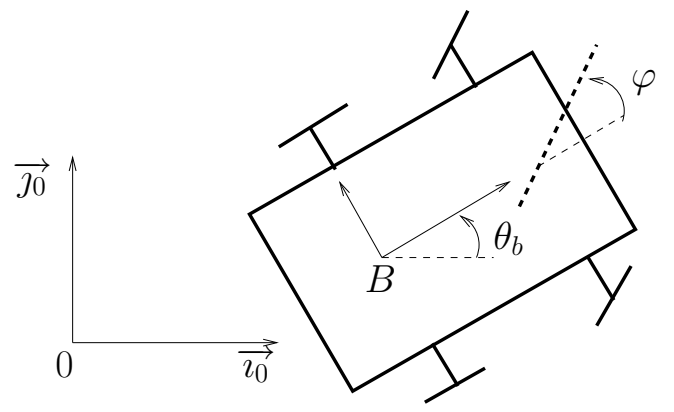

Figure 16: Car-like mobile platform

System (59) can also be written as (compare with (3))

$$
\left\{\begin{array}{l}
\dot{r}_{b}=\bar{R}\left(\theta_{b}\right) u_{b} \\
\dot{\xi}=v_{\xi}
\end{array}\right.
$$

with $u_{b}=\left(v_{b, 1}, 0, v_{b, 1} \xi\right)$. Let us now consider a function

$$
f: \alpha \longmapsto f(\alpha)=\left(\begin{array}{c}
p_{f}(\alpha) \\
\theta_{f}(\alpha) \\
\xi_{f}(\alpha)
\end{array}\right)
$$

from $S^{1} \times S^{1}$ to $S E(2) \times \mathbb{R}$ (i.e. $\alpha=\left(\alpha_{1}, \alpha_{2}\right)$ ). We still define $\bar{r}_{b}(\alpha)$ as in (11), i.e.

$$
\bar{r}_{b}(\alpha):=\left(\begin{array}{c}
p_{b}-R\left(\theta_{b}-\theta_{f}(\alpha)\right) p_{f}(\alpha) \\
\theta_{b}-\theta_{f}(\alpha)
\end{array}\right)=\left(\begin{array}{c}
\bar{p}_{b} \\
\bar{\theta}_{b}
\end{array}\right)
$$

so that Eq. (12) is still valid, i.e.

$$
\dot{\bar{r}}_{b}=\bar{R}\left(\bar{\theta}_{b}\right) \bar{u}_{b}
$$

$\mathrm{RR} \mathrm{n}^{\circ} 5556$ 
with

$$
\bar{u}_{b}:=\left(\begin{array}{cc}
I_{2} & -S p_{f}(\alpha) \\
0 & 1
\end{array}\right)\left(\begin{array}{lll}
\bar{R}\left(\theta_{f}(\alpha)\right) & -\frac{\partial f}{\partial \alpha_{1}}(\alpha) & -\frac{\partial f}{\partial \alpha_{2}}(\alpha)
\end{array}\right)\left(\begin{array}{c}
u_{b} \\
\dot{\alpha}_{1} \\
\dot{\alpha}_{2}
\end{array}\right)
$$

Since $u_{b}=\left(v_{b, 1}, 0, v_{b, 1} \xi\right)$, the previous equation can be written as

$$
\bar{u}_{b}:=\left(\begin{array}{cc}
I_{2} & -S p_{f}(\alpha) \\
0 & 1
\end{array}\right) H(\alpha)\left(\begin{array}{c}
v_{b, 1} \\
\dot{\alpha}_{1} \\
\dot{\alpha}_{2}
\end{array}\right)+\left(\begin{array}{cc}
I_{2} & -S p_{f}(\alpha) \\
0 & 1
\end{array}\right)\left(\begin{array}{c}
0 \\
0 \\
v_{b, 1}\left(\xi-\xi_{f}(\alpha)\right)
\end{array}\right)
$$

with

$$
H(\alpha):=\left(\begin{array}{crr}
\cos \theta_{f}(\alpha) & -\frac{\partial x_{f}}{\partial \alpha_{1}}(\alpha) & -\frac{\partial x_{f}}{\partial \alpha_{2}}(\alpha) \\
\sin \theta_{f}(\alpha) & -\frac{\partial y_{f}}{\partial \alpha_{1}}(\alpha) & -\frac{\partial y_{f}}{\partial \alpha_{2}}(\alpha) \\
\xi_{f}(\alpha) & -\frac{\partial \theta_{f}}{\partial \alpha_{1}}(\alpha) & -\frac{\partial \theta_{f}}{\partial \alpha_{2}}(\alpha)
\end{array}\right)
$$

Let us recall that $\xi_{f}$ denotes the last component of the function $f$. Since $\dot{\xi}=v_{\xi}$, the following choice of the steering wheel angular velocity:

$$
v_{\xi}:=-k\left(\xi-\xi_{f}(\alpha)\right)+\dot{\xi}_{f}(\alpha) \quad(k>0)
$$

yields the exponential stabilization of $\xi-\xi_{f}(\alpha)$ to zero. Let us thus assume from now on that $\xi-\xi_{f}(\alpha)$ is identically equal to zero. We deduce from (61) that (compare with (14)):

$$
\bar{u}_{b}=\left(\begin{array}{cc}
I_{2} & -S p_{f}(\alpha) \\
0 & 1
\end{array}\right) H(\alpha)\left(\begin{array}{c}
v_{b, 1} \\
\dot{\alpha}_{1} \\
\dot{\alpha}_{2}
\end{array}\right)=\bar{H}(\alpha)\left(\begin{array}{c}
v_{b, 1} \\
\dot{\alpha}_{1} \\
\dot{\alpha}_{2}
\end{array}\right)
$$

so that the frame $\mathcal{F}_{\bar{b}}(\alpha)$ associated with $\bar{r}_{b}$ can be viewed as an omnidirectional frame provided that the matrix $H(\alpha)$ given by (62) is invertible. Let us momentarily assume that this property holds. Then, since the control approach presented in Section 3 only relies on the fact that the frame $\mathcal{F}_{\bar{b}}(\alpha)$ is omnidirectional (and not on the characteristics of the mobile platform), the feedback laws $u_{q}$ and $\bar{u}_{b}$ given by (17) and (37) can again be used to yield the same closed-loop dynamics for the manipulation task $e_{m}$ and the secondary $\operatorname{cost} h_{s}$ as those derived for the unicycle-like platform ${ }^{2}$. The control inputs for the platform (i.e. $v_{b, 1}$ and $v_{\xi}$ ) are then computed according to (63) and (64), instead of (14) in the case of a unicycle-like platform.

\footnotetext{
${ }^{2}$ Note however that, when $K$ in (37) is chosen according to (36), the dynamics of $h_{s}$ can be slightly different for the two types of platforms since the associated matrices $\bar{H}(\alpha)$ are not the same.
} 
There remains to consider the issue of the invertibility of $H(\alpha)$ (for any $\alpha$ ). This corresponds to the transversality property of the function $f$, as defined in [9]. A family of transverse functions for the car has been proposed in [11]:

Lemma 1 [11] For any $\varepsilon>0$, and $\eta_{1}, \eta_{2}, \eta_{3}$ such that

$$
\eta_{1}, \eta_{2}, \eta_{3}>0, \quad \text { and } \quad 6 \eta_{2} \eta_{3}>8 \eta_{3}+\eta_{1} \eta_{2}
$$

the function $f$ defined by

$$
f(\alpha)=\left(\begin{array}{c}
\bar{f}_{1}(\alpha) \\
\bar{f}_{4}(\alpha) \\
\arctan \left(\bar{f}_{3}(\alpha)\right) \\
\bar{f}_{2}(\alpha) \cos ^{3} f_{3}(\alpha)
\end{array}\right)
$$

with $\bar{f}: S^{1} \times S^{1} \longrightarrow \mathbb{R}^{4}$ given by

$$
\bar{f}(\alpha)=\left(\begin{array}{c}
\varepsilon\left(\sin \alpha_{1}+\eta_{2} \sin \alpha_{2}\right) \\
\varepsilon \eta_{1} \cos \alpha_{1} \\
\varepsilon^{2}\left(\frac{\eta_{1} \sin 2 \alpha_{1}}{4}-\eta_{3} \cos \alpha_{2}\right) \\
\varepsilon^{3}\left(\eta_{1} \frac{\sin ^{2} \alpha_{1} \cos \alpha_{1}}{6}-\frac{\eta_{2} \eta_{3} \sin 2 \alpha_{2}}{4}-\eta_{3} \sin \alpha_{1} \cos \alpha_{2}\right)
\end{array}\right)
$$

satisfies the transversality condition $\operatorname{det} H(\alpha) \neq 0 \forall \alpha$, where $H(\alpha)$ is defined by (62).

Let us remark that for the family of functions defined in this lemma, the norm of $\bar{f}$ (like the distance from $r_{b}$ to $\bar{r}_{b}$ ) tends to zero as $\varepsilon$ tends to zero. Therefore, the distance from $\mathcal{F}_{b}$ to the companion frame $\mathcal{F}_{\bar{b}}(\alpha)$ can also be rendered arbitrarily small in this case, via the choice of the parameter $\varepsilon$.

\section{Conclusion}

A framework for the feedback control of mobile manipulators, with a focus on motion coordination between the manipulator and the supporting mobile platform, has been proposed. It applies to omnidirectional platforms as well as to nonholonomic ones. In this respect, the concept of omnidirectional companion frame associated with the body of the mobile platform, and derived from the transverse function approach [10], is instrumental. It basically allows to control any nonholonomic platform as if it were omnidirectional.

From there, the control design methodology is influenced by the complementarity of the roles played by the manipulator and the mobile platform. The idea is that a

$\mathrm{RR} \mathrm{n}^{\circ} 5556$ 
manipulation task, which the manipulator is in charge of performing independently of the mobile platform's motion, is first specified in the form of a task-function which has to be regulated to zero. This function must also incorporate terms which allow to unconditionally avoid collisions with the manipulator's joint limits, as well as "regularizing" terms whose role is to ensure that the associated control problem is well-conditionned (in the sense of the "task-admissibility" defined in [14]). As for the platform's motion, it is determined by a secondary cost-function that the control attempts to minimize (a technique reminiscent of a method used for taskredundancy). This function, which depends on the mobile platform's situation, can be a weighted sum of several more elementary functions, each of them corresponding to a specific, sometimes antagonistic, objective. The fact that the exact minimization of this function is often unachievable is not necessarily penalizing because the precise positioning of the mobile platform is usually not required in practice. From these general design rules, many motion coordination strategies can be worked out. We have tried to illustrate some of them via two target tracking examples and simulation results. Finally, we wish to mention that this work has been conducted with the hope of attracting the attention of fellow researchers on a broad and rich subject, still largely open.

\section{References}

[1] G. Artus, P. Morin, and C. Samson. Control of a maneuvering mobile robot by transverse functions. In Symp. on Advances in Robot Kinematics (ARK), pages 459-468, 2004.

[2] B. Bayle, J.-Y. Fourquet, F. Lamiraux, and M. Renaud. Kinematic control of wheeled mobile manipulators. In International Conference on Intelligent Robots and Systems (IROS), pages 1572-1577, 2002.

[3] B. Bayle, J.-Y. Fourquet, and M. Renaud. Génération de mouvements des manipulateurs mobiles. Journal Européen des Systèmes Automatisés (JESA), $35: 809-845,2001$.

[4] R.W. Brockett. Asymptotic stability and feedback stabilization. In R.W. Brock-

ett, R.S. Millman, and H.J. Sussmann, editors, Differential Geometric Control Theory. Birkauser, 1983. 
[5] J.P. Desai, C.C. Wang, M. Zefran, and V. Kumar. Motion planning for multiple mobile manipulators. In IEEE Conf. on Robotics and Automation (ICRA), pages 2073-2078, 1996.

[6] G.W. Haynes and H. Hermes. Nonlinear controllability via Lie theory. SIAM Journal on Control, 8:450-460, 1970.

[7] J.-K. Lee and H. S. Cho. Mobile manipulator motion planning for multiple tasks using global optimization approach. Journal of intelligent and robotic systems, 18:169-190, 1997.

[8] D.A. Lizárraga. Obstructions to the existence of universal stabilizers for smooth control systems. Mathematics of Control, Signals, and Systems, 16:255-277, 2004 .

[9] P. Morin and C. Samson. A characterization of the Lie algebra rank condition by transverse periodic functions. SIAM Journal on Control and Optimization, 40(4):1227-1249, 2001.

[10] P. Morin and C. Samson. Practical stabilization of driftless systems on Lie groups: the transverse function approach. IEEE Trans. on Automatic Control, 48:1496-1508, 2003.

[11] P. Morin and C. Samson. Trajectory tracking for non-holonomic vehicles: overview and case study. In K. Kozlowski, editor, 4th Inter. Workshop on Robot Motion Control (RoMoCo), pages 139-153, 2004.

[12] R.M. Murray, Z. Li, and S.S. Sastry. A mathematical introduction to robotic manipulation. CRC Press, 1994.

[13] C. Perrier, P. Dauchez, and F. Pierrot. A global approach for motion generation of non-holonomic mobile manipulators. In IEEE Conf. on Robotics and Automation (ICRA), pages 2971-2976, 1998.

[14] C. Samson, M. Leborgne, and B. Espiau. Robot Control: The Task-function Approach. Number 22 in Oxford Engineering. Oxford University Press, 1991.

[15] H. Seraji. A unified approach to motion control of mobile manipulators. The International Journal of Robotics Research, 17:107-118, 1998.

$\mathrm{RR} \mathrm{n}^{\circ} 5556$ 
[16] H.J. Sussmann and W. Liu. Limits of highly oscillatory controls and approximation of general paths by admissible trajectories. In IEEE Conf. on Decision and Control (CDC), pages 437-442, 1991.

[17] C.-C. Wang and V. Kumar. Velocity control of mobile manipulators. In IEEE Conf. on Robotics and Automation (ICRA), volume 2, pages 713-718, 1993.

[18] Y. Yamamoto and X. Yun. Coordinating locomotion and manipulation of a mobile manipulator. In IEEE Conf. on Decision and Control (CDC), pages 2643-2648, 1992. 


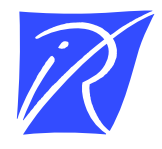

\section{Unité de recherche INRIA Sophia Antipolis 2004, route des Lucioles - BP 93 - 06902 Sophia Antipolis Cedex (France)}

Unité de recherche INRIA Futurs : Parc Club Orsay Université - ZAC des Vignes 4, rue Jacques Monod - 91893 ORSAY Cedex (France)

Unité de recherche INRIA Lorraine : LORIA, Technopôle de Nancy-Brabois - Campus scientifique 615, rue du Jardin Botanique - BP 101 - 54602 Villers-lès-Nancy Cedex (France)

Unité de recherche INRIA Rennes : IRISA, Campus universitaire de Beaulieu - 35042 Rennes Cedex (France)

Unité de recherche INRIA Rhône-Alpes : 655, avenue de l'Europe - 38334 Montbonnot Saint-Ismier (France)

Unité de recherche INRIA Rocquencourt : Domaine de Voluceau - Rocquencourt - BP 105 - 78153 Le Chesnay Cedex (France)

INRIA - Domaine de Voluceau - Rocquencourt, BP 105 - 78153 Le Chesnay Cedex (France)

http://www.inria.fr

ISSN 0249-6399 